\title{
The primary instability of falling films in the presence of soluble surfactants
}

\author{
George Karapetsas $\dagger$ and Vasilis Bontozoglou \\ Department of Mechanical Engineering, University of Thessaly, GR-38334 Volos, Greece
}

(Received 6 February 2013; revised 17 April 2013; accepted 1 June 2013)

We investigate the linear stability of a film flowing down a solid substrate in the presence of soluble surfactants. The Navier-Stokes equations for the liquid motion are considered, together with advection-diffusion equations for the concentrations of the species involved, which include monomers dissolved in the bulk and adsorbed at the liquid-air and at the liquid-substrate interfaces. The adsorption-desorption kinetics of the surfactant at both interfaces is explicitly accounted for. An Orr-Sommerfeld eigenvalue problem is formulated, and solved analytically in the limit of longwave disturbances and numerically for arbitrary wavelength using a finite element method. An extensive parametric study is performed to reveal the role of surfactant solubility and adsorption-desorption kinetics. The results quantify the stabilizing effect of soluble surfactants due to the presence of Marangoni stresses, and indicate that moderately soluble surfactants may be more effective than insoluble ones. Disturbances of finite wavelength are stabilized by more than an order of magnitude, and their detailed behaviour depends in a non-monotonic way on the amount of surfactant and on its solubility and kinetics. The above predictions provide insights for the interpretation of recent experimental findings on the primary instability and on the ensuing unstable dynamics of liquid films doped with soluble surfactants.

Key words: instability, interfacial flows (free surface), Marangoni convection

\section{Introduction}

The formation of waves in thin films flowing down inclined surfaces has attracted the interest of many researchers in the past because of their importance in a broad range of engineering applications. Extensive reviews on the rich dynamics of this system and the main developments in the field are given in Chang (1994), Oron, Davis \& Bankoff (1997) and Craster \& Matar (2009).

It is well known that interfacial instabilities can be significantly affected by the presence of surface-active materials (surfactants). Wave formation in falling films is no exception and this has been known since ancient times. Early experimental studies (e.g. see Emmert \& Pigford 1954; Stirba \& Hurt 1955; Tailby \& Portalski 1961) showed that the addition of even small amounts of surfactant can have a stabilizing influence on the flow, dampening the waves that would otherwise arise on a falling liquid film. The first attempts to investigate the mechanisms that are responsible for the stabilization of the flow were made by Benjamin (1964) and Whitaker (1964) 
following different routes. Whitaker (1964) treated the liquid-air interface as a twodimensional Newtonian fluid and examined the effect of various surface properties such as the surface viscosity, surface elasticity and diffusion of surfactant at the interface from the bulk. He presented numerical solutions of the Orr-Sommerfeld equation, albeit using approximate boundary conditions, and concluded that elasticity, which was due to surface tension gradients, was responsible for the stabilization of the liquid film flowing down a vertical wall below a critical value of the Reynolds number, in contrast to the case of clean liquids where the flow is unstable at all Reynolds numbers. Whitaker \& Jones (1966) and Lin (1970) later presented a perturbation solution of the same problem in the limit of small wavenumbers. Benjamin (1964), on the other hand, considered the interface as a two-dimensional viscoelastic fluid and presented an analytical solution of the Orr-Sommerfeld equation assuming long-wave disturbances. Despite the significant differences between the two approaches, in the case of an insoluble surfactant the analytical prediction was found to be exactly the same, providing an unambiguous confirmation that the elasticity is the mechanism responsible for the stabilization of the flow. An asymptotic analysis for large values of the elasticity parameter by Anshus \& Acrivos (1967) confirmed the findings of the previous works about the existence of a critical Reynolds number and also showed that the presence of surfactants results in the decrease of the growth rate and increase of the wavelength of the most unstable mode.

The theoretical results by Whitaker (1964) also suggested that the wave velocity should increase with increasing elasticity of the interface. However, the experimental work of Strobel \& Whitaker (1969) indicated that the free-surface velocity actually decreases for increased surfactant concentration and this was later confirmed experimentally and theoretically by Cerro \& Whitaker (1971). The latter work also noted that surface velocity depends strongly on the surface elasticity and is only mildly affected by surfactant diffusivity and interfacial mass transport.

More recently the linear stability for vertical film flows with diffusion to the surface and desorption of the surfactant to the gas phase was also examined by Ji \& Setterwall (1994). They were mostly interested in cases where surface instabilities could be enhanced by the presence of surfactants and, for the purposes of their analysis, they chose to ignore the kinetics of adsorption and assumed that there is equilibrium at the interface. Enhancement of instability was also shown by Yiantsios \& Higgins (2010) to be possible under conditions for evaporating thin films in the presence of non-volatile soluble surfactants. Shkadov, Velarde \& Shkadova (2004) used lubrication theory to derive a reduced-order model and examined the linear stability of a similar system taking into account the sorption kinetics at the interface. They were able to recognize four new Marangoni-driven modes and it was shown that the observed modes depend significantly on the sorption kinetics and much less on the equation of state for surface tension.

The effect of insoluble surfactants on the linear stability of a film flowing down a corrugated wall in the limit of vanishing Reynolds number was examined by Pozrikidis (2003). Subsequently, Blyth \& Pozrikidis (2004) presented a numerical solution of the Orr-Sommerfeld eigenvalue problem for finite Reynolds numbers of a film laden with insoluble surfactant flowing on an inclined plane and demonstrated the occurrence of the usual interfacial mode along with a new mode associated with the spatial variation of the surfactant concentration. The same problem was also studied by Pereira \& Kalliadasis (2008) who presented a systematic analysis of the Orr-Sommerfeld problem of the full Navier-Stokes and concentration equations and also investigated the nonlinear dynamics in the unstable regime. 
Despite the large number of studies on the role of surfactants on film flows and the fact that this is a very old problem, it appears from the above review that the effects of surfactant solubility and adsorption/desorption kinetics on the primary instability and on the unstable dynamics have not yet been adequately addressed. In addition, there is recent experimental activity that points to a non-trivial influence of these parameters. For example, Georgantaki, Vlachogiannis \& Bontozoglou (2012) performed a series of experiments on inclined film flows to study the role of different agents that may have an important effect on the interfacial tension of water. For the purposes of their study, Georgantaki et al. used aqueous solutions of isopropanol (IP) and sodium dodecyl sulphate (SDS) and found that these two agents exhibit a remarkably different influence on the flow. More specifically, it was shown that, in the case of IP solutions, the inlet disturbances turned into solitary humps preceded by capillary ripples, as would be expected for a clean fluid with similar surface tension as the IP solution. On the contrary, when SDS solutions were used, the dominant structures were sinusoidal travelling waves of small amplitude. These structures were found to be extremely stable for a wide range of frequencies and up to high Reynolds numbers. Also, with respect to the primary instability, the same authors observed a strong stabilization of water films by the addition of small amounts of SDS, whereas previous results with IP solutions (Georgantaki et al. 2011) had shown no difference in the critical Reynolds number from that of other clean liquids with the same Kapitza number (the definition of $K a$ is given in $\S 3$ below).

Substances IP and SDS have very different properties, and in a sense represent two extreme examples of soluble agents that modify the surface properties of water. IP is very soluble and decreases significantly the surface tension of its solutions. However, it does not show any particular affinity for the liquid-air interface, and thus may not formally be classified as a surfactant. In contrast, SDS exhibits strong partitioning between the liquid-air interface and the bulk. The behaviour of these two agents brackets a whole range of additives that are moderately soluble and show an increasing affinity for the interface, and a theoretical treatment that recovers both limits is evidently welcome. The mechanisms that are responsible for the transition from the behaviour of IP to that of SDS are not fully understood, and it is speculated that they may be an outcome of the different solubility and/or sorption kinetics.

The scope of our study is to reveal these mechanisms and to this end we examine in detail the linear stability of a film laden with a non-volatile, soluble surfactant flowing down an inclined plane. We perform a systematic analysis of the Orr-Sommerfeld eigenvalue problem of the full Navier-Stokes and concentration equations, taking into account mass exchange by diffusion and convection between the bulk and the interfaces, as well as the effect of sorption kinetics along the liquid-air interface and along the substrate. We focus on surfactant concentrations below the critical micelle concentration (CMC) and as a result, we ignore for the moment possible effects of intrinsic surface viscosity (Fruhner, Wantke \& Lunkenheimer 1999), which might be non-negligible at higher concentrations.

The paper is organized as follows. In $\S \S 2$ and 3 we describe the details of our model and the governing equations. The linear stability analysis is performed in $\S 4$, and consists of an analytical solution in the limit of disturbances with very small wavenumber and a numerical solution for arbitrary wavenumbers. The results are presented and discussed in $\S 5$, followed by concluding remarks in $\S 6$. 


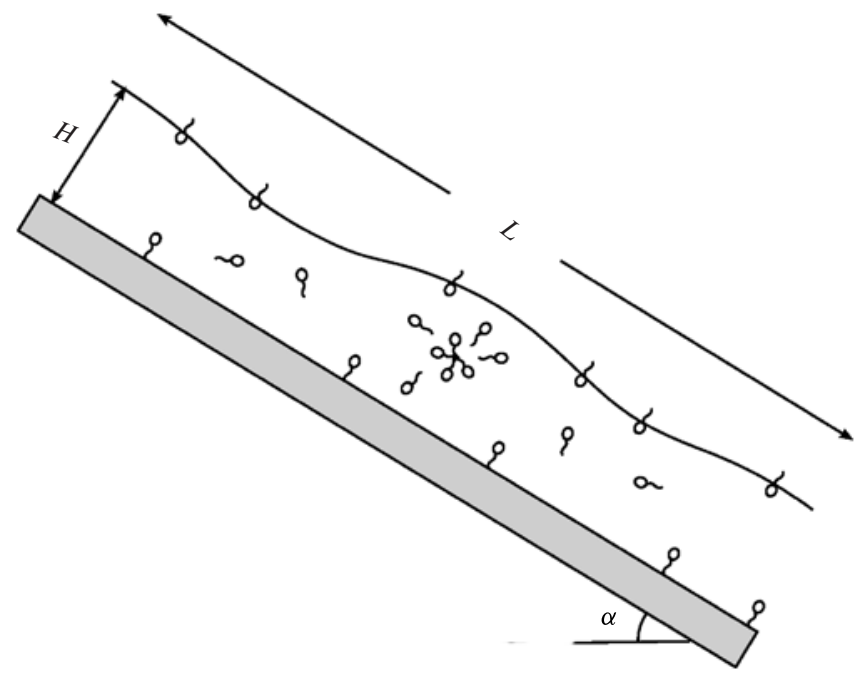

FIGURE 1. Schematic of a falling film in the presence of soluble surfactants.

\section{Problem formulation}

We consider the dynamics of liquid films flowing along an infinite planar wall, inclined at an angle $\alpha$ with the horizontal plane (see figure 1). The film is laden with a soluble, non-volatile surfactant which may adsorb at the liquid-air interface altering the surface tension, or it can exist in the bulk in the form of monomers or it may adsorb at the liquid-solid interface. The fluid is Newtonian with density $\rho$, kinematic viscosity $v$ and surface tension $\sigma$; the density and kinematic viscosity are considered constant whereas the surface tension depends on the interfacial concentration of the surfactant.

In order to model two-dimensional dynamics, we use a Cartesian coordinate system $(x, z)$, with $x$ pointing in the streamwise and $z$ in the cross-stream direction. The velocity field is $\boldsymbol{u}=(u, w)$, where $u$ and $w$ are the velocity components in the streamwise and the cross-stream direction. The liquid-air interface is located at $z=h(x, t)$ and the liquid-solid interface at $z=0$. The flow is incompressible and governed by the momentum and mass conservation equations:

$$
\begin{gathered}
\boldsymbol{u}_{t}+\boldsymbol{u} \cdot \nabla \boldsymbol{u}+\frac{1}{\rho} \nabla p-v \nabla^{2} \boldsymbol{u}-\boldsymbol{g}=0, \\
\nabla \cdot \boldsymbol{u}=0
\end{gathered}
$$

where $p$ is the pressure, $\nabla$ is the gradient operator and $g=g(\sin \alpha,-\cos \alpha)$. Unless stated otherwise, the subscripts denote partial differentiation with respect to $x, z$ and time $t$.

Solutions of (2.1) and (2.2) are obtained subject to the following boundary conditions. Along the free surface, the velocity field satisfies the local force balance between normal and viscous stresses in the liquid. Taking the components of this force balance tangential and normal to the free surface $(z=h(x, t))$ we obtain

$$
\begin{gathered}
\boldsymbol{n} \cdot \boldsymbol{\tau} \cdot \boldsymbol{t}=\boldsymbol{t} \cdot \nabla_{s} \sigma, \\
\boldsymbol{n} \cdot \boldsymbol{\tau} \cdot \boldsymbol{n}=-p_{\text {air }}+2 \kappa \sigma,
\end{gathered}
$$


where $\boldsymbol{n}=\left(-h_{x}, 1\right) /\left(1+h_{x}^{2}\right)^{1 / 2}$ and $\boldsymbol{t}=\left(1, h_{x}\right) /\left(1+h_{x}^{2}\right)^{1 / 2}$ denote respectively the outward unit normal and unit tangential vectors on the interface; $2 \kappa$ is the mean curvature of the free surface and $\nabla_{s}$ is the surface gradient operator, defined as

$$
2 \kappa=-\nabla_{s} \cdot \boldsymbol{n}, \quad \nabla_{s}=(\boldsymbol{I}-\boldsymbol{n n}) \cdot \nabla,
$$

and $\boldsymbol{\tau}$ is the total stress tensor,

$$
\boldsymbol{\tau}=-p \boldsymbol{I}+\rho v\left(\nabla \boldsymbol{u}+(\nabla \boldsymbol{u})^{\mathrm{T}}\right),
$$

where $\boldsymbol{I}$ is the identity tensor.

In addition, along the moving interface $(z=h(x, t))$ we impose the kinematic boundary condition,

$$
h_{t}+u h_{x}=w .
$$

At the liquid-solid interface $(z=0)$, the usual no-slip, no-penetration conditions are imposed,

$$
u=0, \quad w=0 .
$$

To account for the presence of soluble surfactants, we utilize the surfactant kinetic model of Edmonstone, Craster \& Matar (2006) and Karapetsas, Craster \& Matar $(2011 a, b)$ that allows in general for two surfactant species in the bulk (monomers and micelle aggregates) and one at each interface. For the present study we consider surfactant concentrations below the critical micelle concentration and thereby we will not account for the presence of micelles but will only consider monomers that may live in the bulk with concentration, $c$. The concentrations of surfactant adsorbed at the liquid-air and liquid-solid interface are denoted respectively by $c_{a}$ and $c_{s}$, and they are connected to the local bulk concentration of monomer according to the following kinetic laws:

$$
S_{a}+c \underset{k_{2}}{\stackrel{k_{1}}{\rightleftharpoons}} c_{a}
$$

and

$$
S_{s}+c \underset{k_{4}}{\stackrel{k_{3}}{\rightleftharpoons}} c_{s} .
$$

The terms $S_{i}$ ( $i=a, s$ for the interface and the substrate) represent the fraction of the respective area that is not covered with monomer, and is thus available for adsorption. They are defined as

$$
S_{i}=1-\frac{c_{i}}{c_{i \infty}} \quad(i=a, s),
$$

where $c_{i \infty}(i=a, s)$ are respectively the surfactant concentrations at the liquid-air interface and at the substrate at maximum packing. Note that each 'reaction' used for this model is characterized by a rate constant $k_{i}$, with $i=1,2,3,4$. The limitation set by the above kinetic laws on the amount of monomer that can be adsorbed at each boundary leads to Langmuir adsorption isotherms (Edwards, Brenner \& Wasan 1991). Indeed, equating adsorption and desorption rates, and taking $c$ as the bulk concentration close to the respective interface, we obtain the following expressions for the interfacial concentrations:

$$
\frac{c_{a}}{c_{a \infty}}=\frac{c}{c+\left(k_{2} c_{a \infty} / k_{1}\right)}, \quad \frac{c_{s}}{c_{s \infty}}=\frac{c}{c+\left(k_{4} c_{s \infty} / k_{3}\right)} .
$$


In the general case away from equilibrium, we use the above kinetic laws to generate the following fluxes that determine how the surfactant transfers between the different phases:

$$
\begin{gathered}
J_{b a}=\left.k_{1} c\right|_{z=h}\left(1-\frac{c_{a}}{c_{a \infty}}\right)-k_{2} c_{a}, \\
J_{b s}=\left.k_{3} c\right|_{z=0}\left(1-\frac{c_{s}}{c_{s \infty}}\right)-k_{4} c_{s},
\end{gathered}
$$

where $J_{b a}$ and $J_{b s}$ denote the flux of monomers from the bulk to the liquid-air and liquid-solid interface, respectively.

The behaviour of the various surfactant species is modelled by the following advection-diffusion equations:

$$
\begin{gathered}
c_{a, t}+\nabla_{s} \cdot\left(\boldsymbol{u}_{s} c_{a}\right)+c_{a}\left(\nabla_{s} \cdot \boldsymbol{n}\right)(\boldsymbol{u} \cdot \boldsymbol{n})=D_{a} \nabla_{s}^{2} c_{a}+J_{b a}, \\
c_{t}+\boldsymbol{u} \cdot \nabla c=D_{b} \nabla^{2} c, \\
c_{s, t}=D_{s} \nabla_{s}^{2} c_{s}+J_{b s},
\end{gathered}
$$

where $\boldsymbol{u}_{s}$ is the tangential velocity at the interface defined as $\boldsymbol{u}_{s}=(\boldsymbol{I}-\boldsymbol{n n}) \cdot \boldsymbol{u}$ and $D_{i}(i=a, b, s)$ denote the diffusion coefficients of the monomers at the liquid-air interface, in the bulk and at the substrate, respectively.

For the monomers in the bulk we apply the following boundary conditions along the interface and the substrate:

$$
\begin{aligned}
J_{b a} & =-D_{b}(\boldsymbol{n} \cdot \nabla c)_{z=h}, \\
J_{b s} & =-D_{b}(\boldsymbol{n} \cdot \nabla c)_{z=0} .
\end{aligned}
$$

To complete the description, a constitutive equation that describes the dependence of the interfacial tension on the surfactant concentrations is required. To this end, we use the Sheludko equation of state (Sheludko 1967; Gaver \& Grotberg 1990):

$$
\sigma=\sigma_{c}\left(1+\frac{c_{a}}{c_{a \infty}}\left[\left(\frac{\sigma_{c}}{\sigma_{m}}\right)^{1 / 3}-1\right]\right)^{-3},
$$

where $\sigma_{c}$ and $\sigma_{m}$ are the surface tensions of a surfactant-free fluid and of maximal surfactant concentration, respectively. This model is nonlinear and asymptotes to a minimal surface tension, $\sigma_{m}$, at high concentrations of adsorbed surfactant, which makes it appropriate for use at high surfactant concentrations, approaching the critical micelle concentration.

The total mass of the surfactant added to the liquid film per unit width, $M_{t o t}$, is a conserved quantity, given by

$$
\int_{0}^{L} \int_{0}^{h} c \mathrm{~d} z \mathrm{~d} x+\int_{0}^{L} c_{a} \mathrm{~d} x+\int_{0}^{L} c_{s} \mathrm{~d} x=M_{t o t},
$$

where $L$ is the length of the falling film.

\section{Scaling}

The governing equations and boundary conditions are made dimensionless, using the following scalings:

$$
(\tilde{x}, \tilde{z})=(x, z) / H, \quad \tilde{h}=h / H, \quad \tilde{t}=t U / H, \quad \tilde{\boldsymbol{u}}=\boldsymbol{u} / U,
$$




$$
\begin{aligned}
& p=p_{a i r}+\rho g H \sin \alpha \tilde{p}, \quad\left(\tilde{c}_{a}, \tilde{c}_{s}, \tilde{c}\right)=\left(\frac{c_{a}}{c_{a \infty}}, \frac{c_{s}}{c_{s \infty}}, \frac{c}{c_{c m c}}\right), \\
& \left(\tilde{J}_{b a}, \tilde{J}_{b s}\right)=\left(\frac{J_{b a}}{c_{a \infty}}, \frac{J_{b s}}{c_{s \infty}}\right) \frac{H}{U}, \quad \tilde{\sigma}=\frac{\sigma}{\sigma_{c}}, \quad \tilde{M}_{t o t}=\frac{M_{t o t}}{H L c_{c m c}},
\end{aligned}
$$

where $H=(3 v Q /(g \sin \alpha))^{1 / 3}$ is the height of the Nusselt flat film, $Q$ is the imposed flow rate, $U$ is the corresponding interfacial velocity, $U=g H^{2} \sin \alpha /(2 v)$, and $c_{c m c}$ is the critical micelle concentration. The tildes denote dimensionless variables and are henceforth suppressed. Using this scaling, three dimensionless numbers emerge: the Reynolds, Weber and surface Péclet numbers, respectively defined

$$
R e=\frac{\chi}{2} \sin \alpha, \quad W e=\frac{K a}{\chi^{2 / 3} \sin \alpha}, \quad P e_{i}=\operatorname{ReSc}_{i} \quad(i=a, s, b, m),
$$

where

$$
\chi=\frac{g H^{3}}{v^{2}}, \quad K a=\frac{\sigma_{c}}{\rho g^{1 / 3} v^{4 / 3}}, \quad S c_{i}=\frac{v}{D_{i}} \quad(i=a, b, s),
$$

correspond to a modified Reynolds number, the Kapitza number and the Schmidt number, respectively. The parameterization shown in (3.5) is advantageous for the study of our system since the modified Reynolds number, $\chi$, is the only parameter in (3.5) that depends on the film height, which is a measure of the flow rate, whereas $K a$ and $S c_{i}(i=a, b, s)$ depend only on material properties. For a given liquid and surfactant the only free parameters are $\chi$ and $\alpha$ while the rest remain constant. This is very useful for our parametric study, and thus parameterization according to (3.5) is preferred over that of (3.4), and will be used extensively in the discussion of the results.

Substitution of these scalings into the momentum and mass conservation governing equations yields

$$
\begin{gathered}
\operatorname{Re}\left(u_{t}+u u_{x}+w u_{z}\right)+2 p_{x}-u_{x x}-u_{z z}-2=0, \\
\operatorname{Re}\left(w_{t}+u w_{x}+w w_{z}\right)+2 p_{z}-w_{x x}-w_{z z}+2 \cot \alpha=0, \\
u_{x}+w_{z}=0 .
\end{gathered}
$$

The behaviour of the surfactant monomers in the bulk is governed by the following dimensionless equation:

$$
c_{t}+u c_{x}+w c_{z}=P e_{b}^{-1}\left(c_{x x}+c_{z z}\right) .
$$

The above set of equations is subject to the following boundary equations along the interface $(z=h(x, t))$ :

$$
\begin{gathered}
-4 u_{x} h_{x}+\left(u_{z}+w_{x}\right)\left(1-h_{x}^{2}\right)=2 W e \sigma_{x} \sqrt{1+h_{x}^{2}}, \\
p+\frac{u_{x}\left(1-h_{x}^{2}\right)+\left(u_{z}+w_{x}\right) h_{x}}{1+h_{x}^{2}}=-W e \sigma \frac{h_{x x}}{\left(1+h_{x}^{2}\right)^{3 / 2}}, \\
c_{a, t}+u c_{a, x}+\frac{c_{a}+u h_{x}=w,}{1+h_{x}^{2}}\left[\left(u_{x}+h_{x} w_{x}\right)+h_{x}\left(u_{z}+h_{x} w_{z}\right)\right] \\
=\frac{1}{P e_{c a} \sqrt{1+h_{x}^{2}}}\left[\frac{c_{a, x}}{\sqrt{1+h_{x}^{2}}}\right]_{x}+J_{b a},
\end{gathered}
$$




$$
\frac{h_{x} c_{x}-c_{z}}{P e_{b} \sqrt{1+h_{x}^{2}}}=\beta_{a} J_{b a}
$$

and along the wall $(z=0)$

$$
\begin{gathered}
u=w=0, \\
c_{s, t}=P e_{c s}^{-1} c_{s, x x}+J_{b s}, \\
\frac{c_{z}}{P e_{b}}=\beta_{s} J_{b s} .
\end{gathered}
$$

The dimensionless expressions for the fluxes are

$$
\begin{gathered}
J_{b a}=k_{a}\left(\left.R_{a} c\right|_{z=h}\left(1-c_{a}\right)-c_{a}\right), \\
J_{b s}=k_{s}\left(\left.R_{s} c\right|_{z=0}\left(1-c_{s}\right)-c_{s}\right),
\end{gathered}
$$

where the dimensionless parameters $\beta_{i}, k_{i}$ and $R_{i}(i=a, s)$ are given by

$$
\begin{gathered}
\beta_{a}=\frac{c_{a \infty}}{H c_{c m c}}, \quad \beta_{s}=\frac{c_{s \infty}}{H c_{c m c}}, \\
k_{a}=\frac{k_{2} H}{U}, \quad k_{s}=\frac{k_{4} H}{U}, \quad R_{a}=\frac{k_{1} c_{c m c}}{k_{2} c_{a \infty}}, \quad R_{s}=\frac{k_{3} c_{c m c}}{k_{4} c_{s \infty}} .
\end{gathered}
$$

The dimensionless form of the Sheludko equation of state for the surface tension is given by

$$
\sigma=\left[1+c_{a}\left(\Sigma^{1 / 3}-1\right)\right]^{-3},
$$

where $\Sigma=\sigma_{c} / \sigma_{m}$. One additional dimensionless parameter will appear in the next section, as a result of the linearization of the equation of state around the base flow. This parameter, the surface elasticity $E_{o}$, is related to the derivative of surface tension along the interface (equation (3.10)) and is formally defined by (4.24).

At this point, it is instructive to attach physical significance to the various dimensionless parameters just defined, and to draw attention to the ones that will be used more extensively in the discussion of the results. Terms $\beta_{i}(i=a, s)$ are ratios of maximum total amounts of surfactant in the different phases. More specifically, they compare the maximum amount that can adsorb on each boundary to the maximum amount that can reside in the bulk as monomer. Terms $R_{i}(i=a, s)$ are ratios of the maximum possible forward and backward reaction rates for each of the adsorption processes. Their physical significance is further clarified by considering local equilibrium: For example, setting the flux in (3.18) equal to zero, results in the expression $c_{a}=R_{a} c /\left(1+R_{a} c\right)$. For $R_{a} \leqslant O(1)$, we observe that $c_{a}<1$ even when $c=1$, i.e. the interface remains unsaturated at the maximum possible concentration of monomer in the bulk (remember that $c$ is non-dimensionalized with $c_{c m c}$, and $c_{a}$ with $\left.c_{a \infty}\right)$. On the contrary, for $R_{a} \gg O(1)$ the interface is practically saturated at bulk concentration well below the critical value for the formation of micelles.

Parameters $\beta_{i}$ and $R_{i}$ may be combined as follows:

$$
\xi_{a}=\beta_{a} R_{a}=\frac{k_{1}}{H k_{2}}, \quad \xi_{s}=\beta_{s} R_{s}=\frac{k_{3}}{H k_{4}} .
$$

Terms $\xi_{i}(i=a, s)$ are ratios of the kinetic constants of the forward and backward reaction for each interface-bulk interaction, and thus provide a direct measure of the surfactant solubility in the bulk liquid (Jensen \& Grotberg 1993). For example, $\xi_{a} \ll 1$ signifies a highly soluble surfactant, whereas for $\xi_{a} \gg 1$ the surfactant is 
virtually insoluble and is trapped at the interface. We note that most of the recent theoretical literature refers to insoluble surfactants, and thus $\xi_{a} \gg 1$ is a useful limit for comparisons.

We will be making extensive use of the solubilities, $\xi_{i}$, in what follows, because some important aspects of the system behaviour are not dictated by the parameters $\beta_{i}$ and $R_{i}$ independently, but only by their combination $\beta_{i} R_{i}$. This is notably the case for the critical Reynolds number and for the concentration for maximum stability (see (4.42) and (5.1) below).

Finally, parameters $k_{i}(i=a, s)$ are ratios of the time scale of convection to the time scale of each of the reverse reactions. For example, $k_{a} \ll 1$ means that the desorption reaction is slow compared to convection, and thus a temporary decrease of the bulk concentration near the interface will not lead to significant desorption. Similar comparison for the forward reaction (e.g. extent of adsorption resulting from a temporary local increase in the bulk concentration) is accomplished using the parameter $k_{a} \beta_{a} R_{a}=k_{a} \xi_{a}$. Thus, an asymmetry in the adsorption-desorption tendency may be dictated by the value of $\xi_{a}$, i.e. the solubility of the surfactant. For example, a sparingly soluble surfactant could have $k_{a}<1$ and $k_{a} \xi_{a}>1$, and as a result, a temporary decrease of the bulk concentration near the interface will not lead to significant desorption whereas a temporary increase will lead to significant adsorption.

\section{Linear stability analysis}

\subsection{Base state}

The set of dimensionless equations and boundary conditions, derived in the previous section, has a trivial solution corresponding to a flat film with uniform surfactant concentration. In the case of a clean fluid, this is also known as the Nusselt flat film solution and its dimensionless form is

$$
h_{o}=1, \quad u_{o}=2 z-z^{2}, \quad w_{o}=0, \quad p_{o}=(1-z) \cot \alpha .
$$

In order for the above solution to remain valid when a soluble surfactant is present, the concentration of all the species should be uniform and at equilibrium

$$
\left(c_{a}, c_{s}, c\right)=\left(c_{a o}, c_{s o}, c_{o}\right),
$$

where $c_{a o}, c_{s o}, c_{o}$ denote the equilibrium values. The equilibrium surface tension of the liquid-air interface,

$$
\sigma_{o}=\left[1+c_{a o}\left(\Sigma^{1 / 3}-1\right)\right]^{-3},
$$

is also uniform and any variation in concentration would result in surface tension gradients which would drive additional flows. At equilibrium the fluxes should be $J_{b a}=J_{b s}=0$. Thus using (3.18) and (3.19) we get

$$
c_{i o}=\frac{R_{i} c_{o}}{1+R_{i} c_{o}} \quad(i=a, s) .
$$

We also know that the total mass of the surfactant is given by the following equation:

$$
c_{o}+\beta_{a} c_{a o}+\beta_{s} c_{s o}=M_{t o t},
$$

which can be solved numerically with respect to $c_{o}$ for a given value of $M_{t o t}$. Below, we have derived an analytical solution for $c_{o}$ and $c_{a o}$, assuming that there is no 

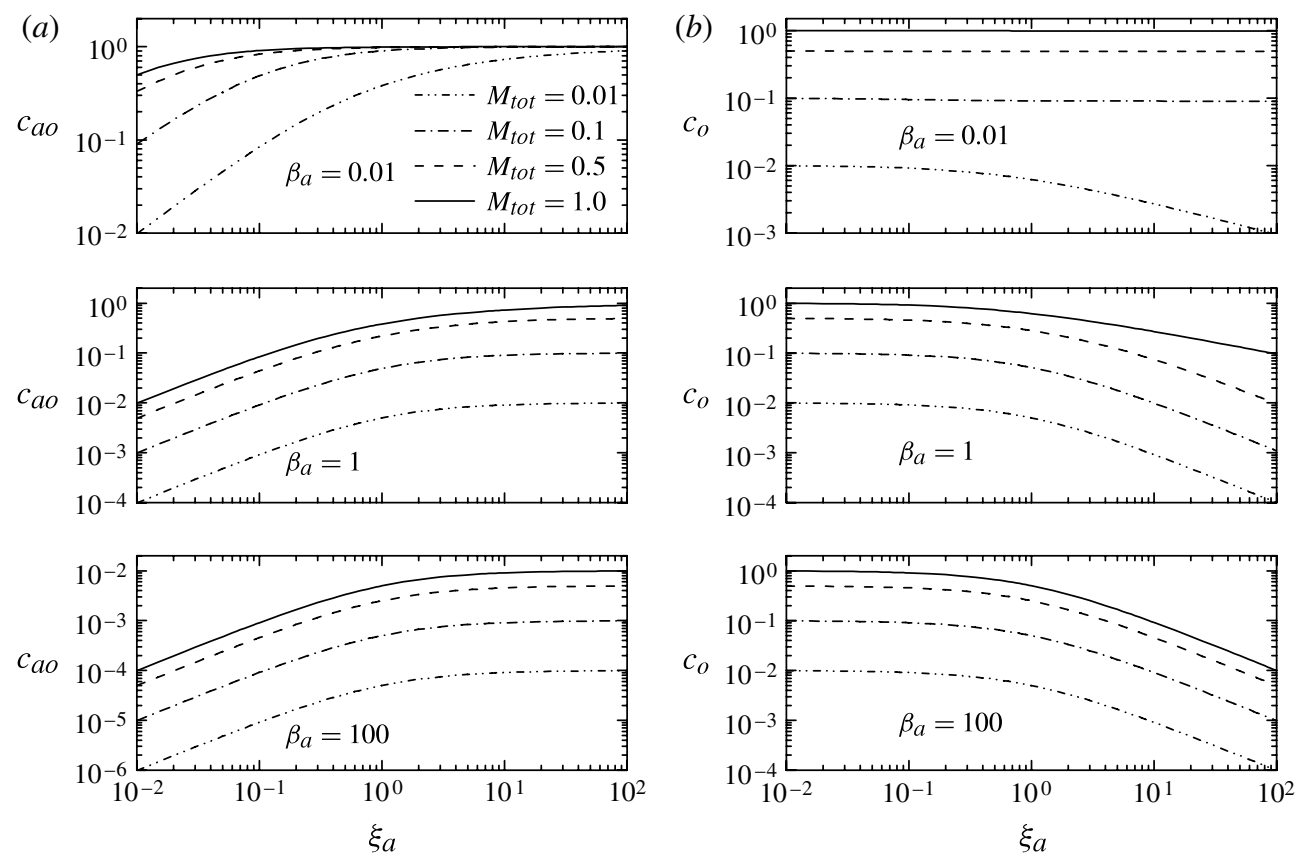

FIGURE 2. Dependence of monomer surfactant concentration at the interface $(a)$ and in the bulk $(b)$ on the parameter $\xi_{a}$ for various values of $\beta_{a}$ and $M_{\text {tot }}$. We assume that surfactant does not adsorb at the substrate $\left(\beta_{s}=R_{s}=0\right)$.

adsorption of surfactant at the substrate, $c_{s o}=0$ :

$$
\begin{gathered}
c_{o}=\frac{-1-\xi_{a}+M_{t o t} \xi_{a} / \beta_{a}+\sqrt{4 M_{t o t} \xi_{a} / \beta_{a}+\left(1+\xi_{a}-M_{t o t} \xi_{a} / \beta_{a}\right)^{2}}}{2 \xi_{a} / \beta_{a}}, \\
c_{a o}=\frac{-1-\xi_{a}+M_{t o t} \xi_{a} / \beta_{a}+\sqrt{4 M_{t o t} \xi_{a} / \beta_{a}+\left(1+\xi_{a}-M_{t o t} \xi_{a} / \beta_{a}\right)^{2}}}{1-\xi_{a}+M_{t o t} \xi_{a} / \beta_{a}+\sqrt{4 M_{t o t} \xi_{a} / \beta_{a}+\left(1+\xi_{a}-M_{t o t} \xi_{a} / \beta_{a}\right)^{2}}} .
\end{gathered}
$$

The above solution is shown graphically in figure 2 as a function of the solubility parameter $\xi_{a}$ and for various values of $\beta_{a}$ and $M_{t o t}$. As expected, we find that for high values of $\xi_{a}$ (low solubility) most of the surfactant is at the interface whereas for low values of $\xi_{a}$ (high solubility) it prefers to be in the bulk.

In the case of an insoluble surfactant, we simply take

$$
c_{a o}=M_{t o t}^{\prime},
$$

where $M_{t o t}^{\prime}=M_{t o t} / \beta_{a}$ and $c_{o}$ is equal to zero.

\subsection{Derivation of linearized equations and boundary conditions}

We perform a linear stability analysis by perturbing the flow around the Nusselt flat film solution. To this end we write all variables as the sum of the base state and a small perturbation,

$$
\phi=\phi_{o}+\bar{\phi},
$$


and linearize the governing equations assuming $\bar{\phi} \ll \phi_{o}$. We end up with the following linearized disturbance equations:

$$
\begin{gathered}
\operatorname{Re}\left(\bar{u}_{t}+\left(2 z-z^{2}\right) \bar{u}_{x}+2(1-z) \bar{w}\right)+2 \bar{p}_{x}-\bar{u}_{x x}-\bar{u}_{z z}=0 \\
\operatorname{Re}\left(\bar{w}_{t}+\left(2 z-z^{2}\right) \bar{w}_{x}\right)+2 \bar{p}_{z}-\bar{w}_{x x}-\bar{w}_{z z}=0 \\
\bar{u}_{x}+\bar{w}_{z}=0 \\
\bar{c}_{t}+\left(2 z-z^{2}\right) \bar{c}_{x}=P e_{b}^{-1}\left(\bar{c}_{x x}+\bar{c}_{z z}\right)
\end{gathered}
$$

and the following boundary conditions along the interface:

$$
\begin{gathered}
-2 \bar{h}+\bar{u}_{z}+\bar{w}_{x}=2 W e \bar{\sigma}_{x}, \\
\bar{p}+\bar{u}_{x}-\bar{h} \cot \alpha=-W e \bar{h}_{x x} \sigma_{o}, \\
\bar{h}_{t}+\bar{h}_{x}=\bar{w} \\
\bar{c}_{a, t}+\bar{c}_{a, x}+c_{a o} \bar{u}_{x}=\frac{\bar{c}_{a, x x}}{P e_{c a}}+\bar{J}_{b a}, \\
\frac{\bar{c}_{z}}{P e_{b}}=-\beta_{a} \bar{J}_{b a} ;
\end{gathered}
$$

and along the wall

$$
\begin{gathered}
\bar{u}=\bar{w}=0, \\
\bar{c}_{s, t}=\frac{\bar{c}_{s, x x}}{P e_{c s}}+\bar{J}_{b s}, \\
\frac{\bar{c}_{z}}{P e_{b}}=\beta_{s} \bar{J}_{b s} .
\end{gathered}
$$

The linearized perturbation fluxes are

$$
\begin{aligned}
& \bar{J}_{b a}=k_{a}\left[R_{a}\left(\left.\bar{c}\right|_{z=h}\left(1-c_{a o}\right)-c_{o} \bar{c}_{a}\right)-\bar{c}_{a}\right], \\
& \bar{J}_{b s}=k_{s}\left[R_{s}\left(\left.\bar{c}\right|_{z=0}\left(1-c_{s o}\right)-c_{o} \bar{c}_{s}\right)-\bar{c}_{s}\right] .
\end{aligned}
$$

It is also worth noting the linearization of the equation of state for the surface tension around the base state, $\sigma-\sigma_{o}=\left(\mathrm{d} \sigma / \mathrm{d} c_{a}\right)_{c_{a o}}\left(c_{a}-c_{a o}\right)$, or $\bar{\sigma}=\left(\mathrm{d} \sigma / \mathrm{d} c_{a}\right)_{c_{a o}} \bar{c}_{a}$. This derivative may be re-framed in terms of the definition of the dimensionless surface elasticity (scaled with surface tension of a clean fluid, $\left.\sigma_{c}\right), E\left(c_{a}\right)$,

$$
E_{o}=E\left(c_{a o}\right)=-\left(\frac{\mathrm{d} \sigma}{\mathrm{d} \ln c_{a}}\right)_{c_{a o}}=-c_{a o}\left(\frac{\mathrm{d} \sigma}{\mathrm{d} c_{a}}\right)_{c_{a o}},
$$

so that perturbations in surface tension are replaced by perturbations in surface concentration:

$$
\bar{\sigma}=-\frac{E_{o}}{c_{a o}} \bar{c}_{a}, \quad \bar{\sigma}_{x}=-\frac{E_{o}}{c_{a o}} \bar{c}_{a, x} .
$$

Using the dimensionless equation of state, surface elasticity is expressed in terms of the surface concentration by

$$
E_{o}=\frac{3 c_{a o}\left(\Sigma^{1 / 3}-1\right)}{\left[1+c_{a o}\left(\Sigma^{1 / 3}-1\right)\right]^{4}} .
$$

Figures 3(a) and 3(b) show respectively the functions $\sigma\left(c_{a o}\right)$ and $E\left(c_{a o}\right)$ and their dependence on the interfacial concentration, $c_{a o}$, and the total amount of surfactant, $M_{\text {tot }}$. It is evident that the Sheludko equation of state exhibits a smooth approach to 

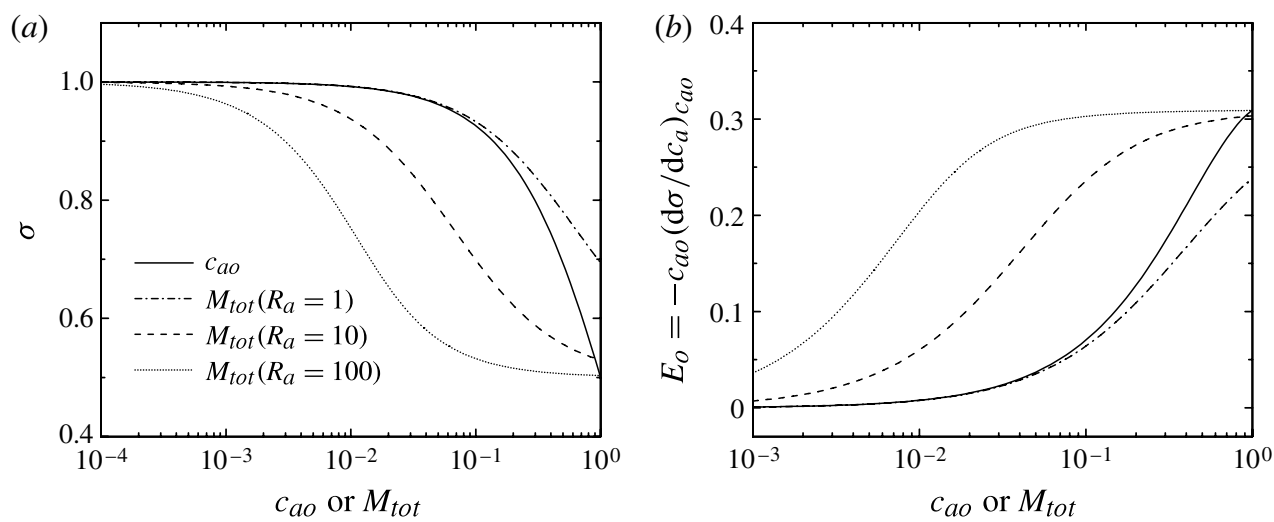

FIGURE 3. Dependence of $(a)$ surface tension and $(b)$ elasticity on the interfacial concentration, $c_{a o}$, and the total amount of surfactant, $M_{t o t}$, for $\beta_{a}=0.01$.

the limiting values for a clear and for a saturated interface, and that, in both these limits, the surface elasticity increases smoothly until it saturates for high amounts of surfactant.

The above set of equations is converted into the Orr-Sommerfeld problem of the linearized Navier-Stokes and concentration equations by introducing the following definition for the stream function:

$$
\bar{u}=\bar{\psi}_{z}, \quad \bar{w}=-\bar{\psi}_{x},
$$

and seeking disturbances in the form of normal modes. To this end, we write all variables in the following way:

$$
\left(\begin{array}{c}
\bar{\psi}(x, z, t) \\
\bar{c}(x, z, t) \\
\bar{c}_{a}(x, t) \\
\bar{c}_{s}(x, t)
\end{array}\right)=\left(\begin{array}{c}
\Psi(z) \\
C(z) \\
C_{a} \\
C_{s}
\end{array}\right) \exp (\lambda t+\mathrm{i} k x),
$$

and end up with the following set of equations

$$
\begin{gathered}
\operatorname{Re}\left[\left(\lambda+\mathrm{i} k\left(2 z-z^{2}\right)\right)\left(\Psi_{z z}-k^{2} \Psi\right)+2 \mathrm{i} k \Psi\right]=\Psi_{z z z z}-2 k^{2} \Psi_{z z}+k^{4} \Psi, \\
P e_{b}\left[\lambda+\mathrm{i} k\left(2 z-z^{2}\right)\right] C=C_{z z}-k^{2} C .
\end{gathered}
$$

These equations are subject to the following boundary conditions along the interface:

$$
\begin{gathered}
\Psi_{z z z}-3 k^{2} \Psi_{z}-\operatorname{Re}(\lambda+\mathrm{i} k) \Psi_{z}=\mathrm{i} k\left[k^{2} W e \sigma_{o}+\cot \alpha\right]\left[\Psi_{z z}+k^{2} \Psi+2 \mathrm{i} k W e \frac{E_{o}}{c_{a o}} C_{a}\right] \\
(\lambda+\mathrm{i} k)\left[\frac{1}{2}\left(\Psi_{z z}+k^{2} \Psi\right)+\mathrm{i} k W e \frac{E_{o}}{c_{a o}} C_{a}\right]+\mathrm{i} k \Psi=0 \\
\left(\lambda+\mathrm{i} k+\frac{k^{2}}{P e_{c a}}\right) C_{a}=-\mathrm{i} k c_{a o} \Psi_{z}+k_{a}\left[R_{a}\left(C\left(1-c_{a o}\right)-c_{o} C_{a}\right)-C_{a}\right] \\
\frac{C_{z}}{P e_{b}}=-\beta_{a} k_{a}\left[R_{a}\left(C\left(1-c_{a o}\right)-c_{o} C_{a}\right)-C_{a}\right]
\end{gathered}
$$


and along the wall

$$
\begin{gathered}
\Psi_{z}=0, \\
\Psi=0, \\
\left(\lambda+\frac{k^{2}}{P e_{c s}}\right) C_{s}=k_{s}\left[R_{s}\left(C\left(1-c_{s o}\right)-c_{o} C_{s}\right)-C_{s}\right], \\
\frac{C_{z}}{P e_{b}}=\beta_{s} k_{s}\left[R_{s}\left(C\left(1-c_{s o}\right)-c_{o} C_{s}\right)-C_{s}\right] .
\end{gathered}
$$

For the case of an insoluble surfactant $\left(\xi_{a} \gg 1\right)$ that does not adsorb to the substrate, the above system readily reduces to the equations presented by Pereira \& Kalliadasis (2008).

\subsection{Analytic predictions by a long-wave expansion}

For the case of a soluble surfactant and for no adsorption of the surfactant at the substrate, it is possible to perform a long-wave expansion of the above set of equations for $k \rightarrow 0$. To this end, we follow a similar procedure as in Pereira \& Kalliadasis (2008) and seek a solution of the form

$$
\left(\begin{array}{c}
\Psi \\
C \\
C_{a}
\end{array}\right)=\left(\begin{array}{c}
\Psi^{0} \\
C^{0} \\
C_{a}^{0}
\end{array}\right) \lambda^{0}+\left(\begin{array}{c}
\Psi^{1} \\
C^{1} \\
C_{a}^{1}
\end{array}\right) \lambda^{1} \mathrm{i} k+\left(\begin{array}{c}
\Psi^{2} \\
C^{2} \\
C_{a}^{2}
\end{array}\right) \lambda^{2} k^{2}+O\left(k^{3}\right) .
$$

Substituting in (4.29)-(4.38) and assuming $C_{s}=\beta_{s}=k_{s}=R_{s}=0$, we expand the resulting equations in series of $k$ and derive the following expression for the eigenvalue of the most unstable mode:

$$
\lambda=-2 \mathrm{i} k+\left(\frac{8}{15} R e-6 M a \frac{\xi_{a}\left(c_{a o}-1\right)^{2}}{3 \xi_{a}\left(c_{a o}-1\right)^{2}+4}-\frac{2}{3} \cot \alpha\right) k^{2},
$$

where $M a$ is the Marangoni number defined as

$$
M a=E_{o} W e=-c_{a o}\left(\frac{\mathrm{d} \sigma}{\mathrm{d} c_{a}}\right)_{c_{a o}} W e=\frac{3 c_{a o}\left(\Sigma^{1 / 3}-1\right)}{\left[1+c_{a o}\left(\Sigma^{1 / 3}-1\right)\right]^{4}} \frac{K a}{\chi^{2 / 3} \sin \alpha} .
$$

The imaginary part of (4.40) gives the expected result that long-wave disturbances travel with phase speed equal to twice the free surface velocity. The real part of (4.40) indicates that the onset of instability occurs when the coefficient of $k^{2}$ vanishes. Therefore the critical Reynolds number is given by the following expression:

$$
R e_{c}=\frac{5}{4} \cot \alpha+\frac{15}{4} M a \frac{3 \xi_{a}\left(c_{a o}-1\right)^{2}}{3 \xi_{a}\left(c_{a o}-1\right)^{2}+4},
$$

where $c_{a o}$ is given by (4.7). We note that (4.42) is implicit in $R e_{c}$ or $\chi_{c}$, because of the appearance of these dimensionless numbers in the definition of $M a$ in (4.41). An explicit expression may be derived only for a vertical film, and is as follows:

$$
\chi_{c}=2 \operatorname{Re}_{c}=\left[\frac{15}{2} E_{o} K a \frac{3 \xi_{a}\left(c_{a o}-1\right)^{2}}{3 \xi_{a}\left(c_{a o}-1\right)^{2}+4}\right]^{3 / 5} .
$$

In the limit of an insoluble surfactant $\left(\xi_{a}=\beta_{a} R_{a} \gg 1\right),(4.42)$ reduces to

$$
R e_{c}=\frac{5}{4} \cot \alpha+\frac{15}{4} M a,
$$


in agreement with the expression given by Pereira \& Kalliadasis (2008). For a clean liquid, $M a=0$, both equations lead to $R e_{c}=(5 / 4) \cot \alpha$, the well known prediction for a Newtonian falling film (Benjamin 1957; Yih 1963). It is interesting to note that this prediction is also recovered in the limit of an interface saturated with a mildly soluble surfactant $\left(c_{a 0} \rightarrow 1, \xi_{a}\right.$ finite), as well as in the limit of a very soluble surfactant $\left(\xi_{a} \ll 1\right)$. The latter prediction is in agreement with the data of Georgantaki et al. (2012), who performed experiments with the highly soluble isopropanol and observed a behaviour representative of a clean liquid with reduced surface tension.

\subsection{Numerical method for arbitrary wavelength}

The Orr-Sommerfeld eigenvalue problem, which consists of (4.29)-(4.38), cannot be solved analytically for arbitrary wavenumbers. The discretization of the governing equations is performed using a finite-element/Galerkin method, and we approximate all the variables through the use of quadratic Lagrangian basis functions $\phi_{i}$. Applying the divergence theorem, the weak form of the governing equations becomes

$$
\begin{gathered}
\int_{0}^{1}\left[\left[\operatorname{Re}\left[\left(\lambda+\mathrm{i} k\left(2 y-y^{2}\right)\right)\left(\Phi_{y}-k^{2} \Psi\right)+2 \mathrm{i} k \Psi\right]+2 k^{2} \Phi_{y}-k^{4} \Psi\right] \phi_{i}\right. \\
\left.+\Phi_{y} \frac{\mathrm{d} \phi_{i}}{\mathrm{~d} y}\right] \mathrm{d} y-\left[\Phi_{y} \phi_{i}\right]_{0}^{1}=0, \\
\int_{0}^{1}\left(\Phi \phi_{i}+\Psi_{y} \frac{\mathrm{d} \phi_{i}}{\mathrm{~d} y}\right) \mathrm{d} y-\left[\Psi_{y} \phi_{i}\right]_{0}^{1}=0, \\
\int_{0}^{1}\left[\left(P e_{b}\left[\lambda+\mathrm{i} k\left(2 y-y^{2}\right)\right]+k^{2}\right) C \phi_{i}+C_{y} \frac{\mathrm{d} \phi_{i}}{\mathrm{~d} y}\right] \mathrm{d} y-\left[C_{y} \phi_{i}\right]_{0}^{1}=0 .
\end{gathered}
$$

Note that the fourth-order partial differential equation for $\Psi$ has been decomposed into two second-order differential equations by introducing a new variable, $\Phi=\Psi_{y y}$. At the edge nodes we impose the boundary conditions that are given by (4.31)-(4.38). The resulting system of algebraic equations is solved numerically with the help of the LAPACK library. The computational domain is discretized using 50 elements in all the computations presented in this paper; numerical checks showed that increasing the number of elements further led to negligible changes.

\section{Discussion}

\subsection{The hydrodynamic instability mode}

In the present section, we will examine the effect of various parameters contained in our model of a soluble surfactant. Thus, we will identify the range of physical phenomena that may be described by this model. In particular, we wish to set the present flow in perspective with the two limiting cases of: $(a)$ a clean liquid; and $(b)$ a liquid doped with an insoluble surfactant. The latter appears to have attracted most of the attention in the recent theoretical literature on film flow with surfactants, and as a result the extent and significance of differences caused by surfactant solubility are presently unclear.

The effect of inertia on the stability of surfactant-laden falling films is demonstrated in figure 4. The values of parameters used are mentioned in the caption, and constitute a reasonable base case. In particular, we assume that the surfactant adsorbs only at the liquid-air interface and not on the solid substrate. Shown in figure 4 is the dispersion relation for increasing values of the modified Reynolds number, $\chi$. Beyond a certain 


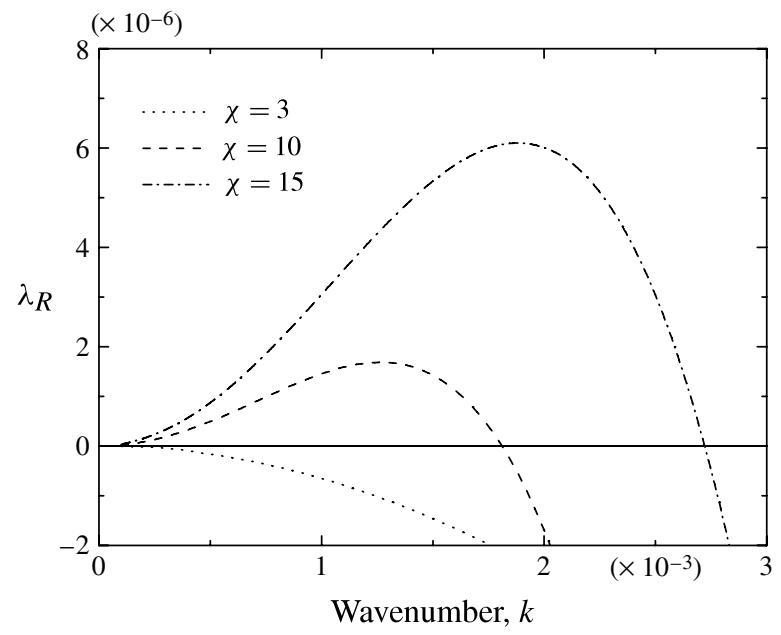

FIgURE 4. Dispersion curves for different values of $\chi$. The rest of the parameters are $\alpha=90^{\circ}, K a=3000, \Sigma=2, M_{\text {tot }}=0.1, \beta_{a}=0.01, R_{a}=1, k_{a}=0.01, \beta_{s}=R_{s}=k_{s}=0$, $S c_{a}=S c_{s}=100$ and $S c_{b}=10$.

(a)

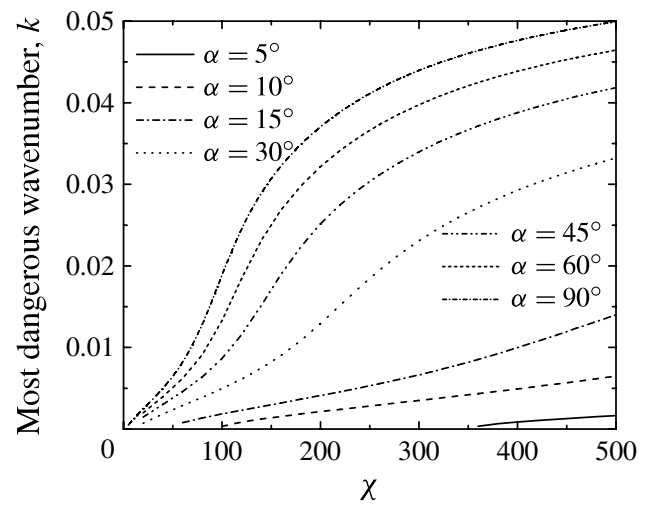

(b)

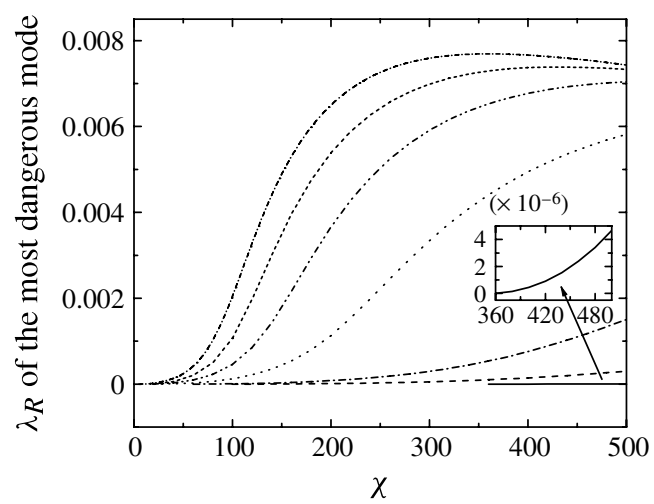

FIGURE 5. Dependence of $(a)$ the wavenumber and $(b)$ the growth rate of the most dangerous mode on $\chi$. The rest of the parameters remain the same as in figure 4 .

threshold, $\chi_{c}$, the flow becomes unstable; below this threshold all modes are stable. For the case shown, the critical $\chi$ is equal to 3.74 .

It is evident from figure 4 that the instability is a long-wave one, and that, for each unstable $\chi$, a wavenumber with maximum growth rate (the most dangerous mode) may be identified. The variation with $\chi$ of the wavenumber and the growth rate of the most dangerous mode at neutral stability is shown respectively for various inclinations in figures $5(a)$ and $5(b)$. The conclusion that the instability is a long-wave one magnifies the significance of the analytic result, (4.42). Thus, we will be making frequent use of it in the subsequent discussion. However, the behaviour of higher wavenumbers is still very important, because these harmonics enter into mode interactions that dictate the nonlinear evolution of the interface. 

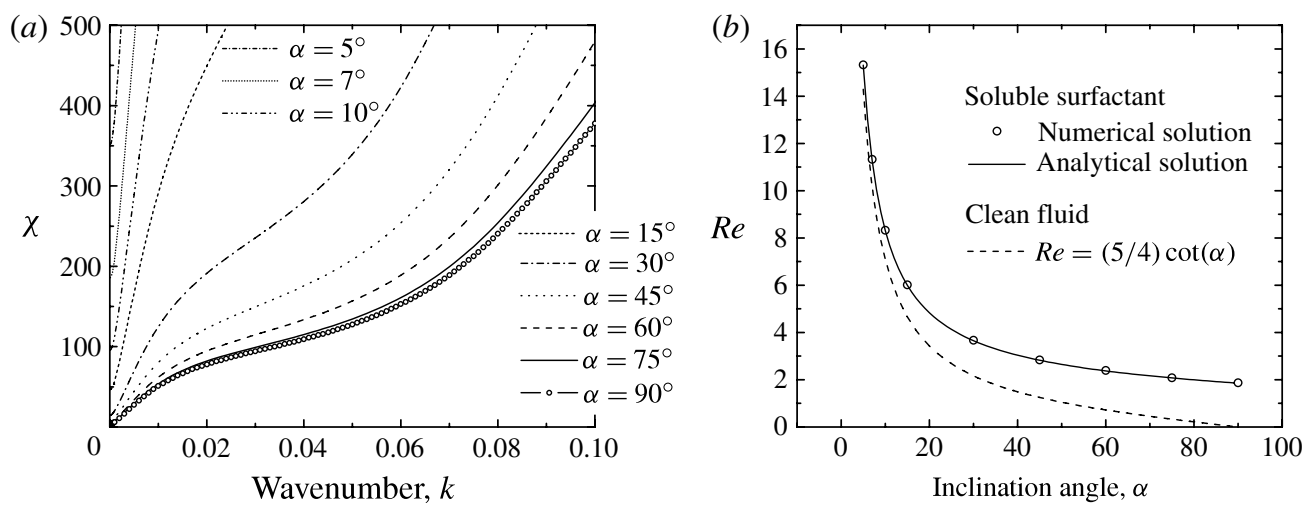

FIgURE 6. (a) Neutral curves for various inclination angles. (b) Dependence of critical Re on the inclination angle; comparison of analytical solution (equation (4.42)) and numerical results for $k=10^{-4}$. The rest of the parameters remain the same as in figure 4 .

To further examine the effect of inclination angle, we present in figure 6(a) neutral stability curves for various inclinations. As expected the most unstable case is for $\alpha=90^{\circ}$. For low values of $\alpha$ the flow remains stable up to rather high values of the modified Reynolds number, $\chi$, and the critical $\chi$ increases abruptly with decreasing wavelength of the perturbations. Figure $6(b)$ presents the dependence of the critical $R e$ on inclination for $k=10^{-4}$. The agreement with the analytical prediction, (4.42), serves as a check of the accuracy of the numerical solution. Also shown in figure $6(b)$ is the prediction for a clean liquid. With decreasing inclination, differences between the two liquids gradually decrease, because the term (5/4) $\cot \alpha$ grows in significance compared to the Marangoni correction. Thus, from here on we concentrate on the most interesting case of a vertical film.

\subsection{Parametric behaviour of the critical conditions}

We start by considering the critical conditions, which correspond to the onset of longwave disturbances, i.e. $k \rightarrow 0$. A question of evident interest is the effect on stability of the total amount of surfactant contained per unit area of the channel. To this end, we present in figure $7(a)$ the critical $\chi_{c}$ as a function of $M_{t o t}$, for three different values of the solubility parameter, $\xi_{a}$. The lines in this figure depict the analytical solution (equation (4.42)) while the points depict the numerical solution, which is in very good agreement with the analytical solution, testifying thus once again to its accuracy.

It is notable that the dependence on $M_{t o t}$ is not monotonic, exhibiting maximum stabilization at an intermediate value $M_{\text {tot, } \max }$, below the CMC. For very small, as well as for large values of $M_{t o t}$, the critical $\chi_{c}$ approaches the limit of the clean fluid. This is somewhat counter-intuitive because one would expect that the more surfactant is present in the system the more stable the system would be. However, we should keep in mind that it is not the amount of surfactant that stabilizes the flow, but actually the Marangoni stresses, which are related to the elasticity of the interface. When the surfactant exhibits significant solubility, the behaviour is further complicated because of two competing effects; with increasing amount of surfactant, the dimensionless elasticity, $E_{o}$, grows as depicted in figure 3. At the same time, the growth in bulk concentration provides higher driving force for mass transfer with the interface, leading to the attenuation of surface tension gradients. As a result, when the 

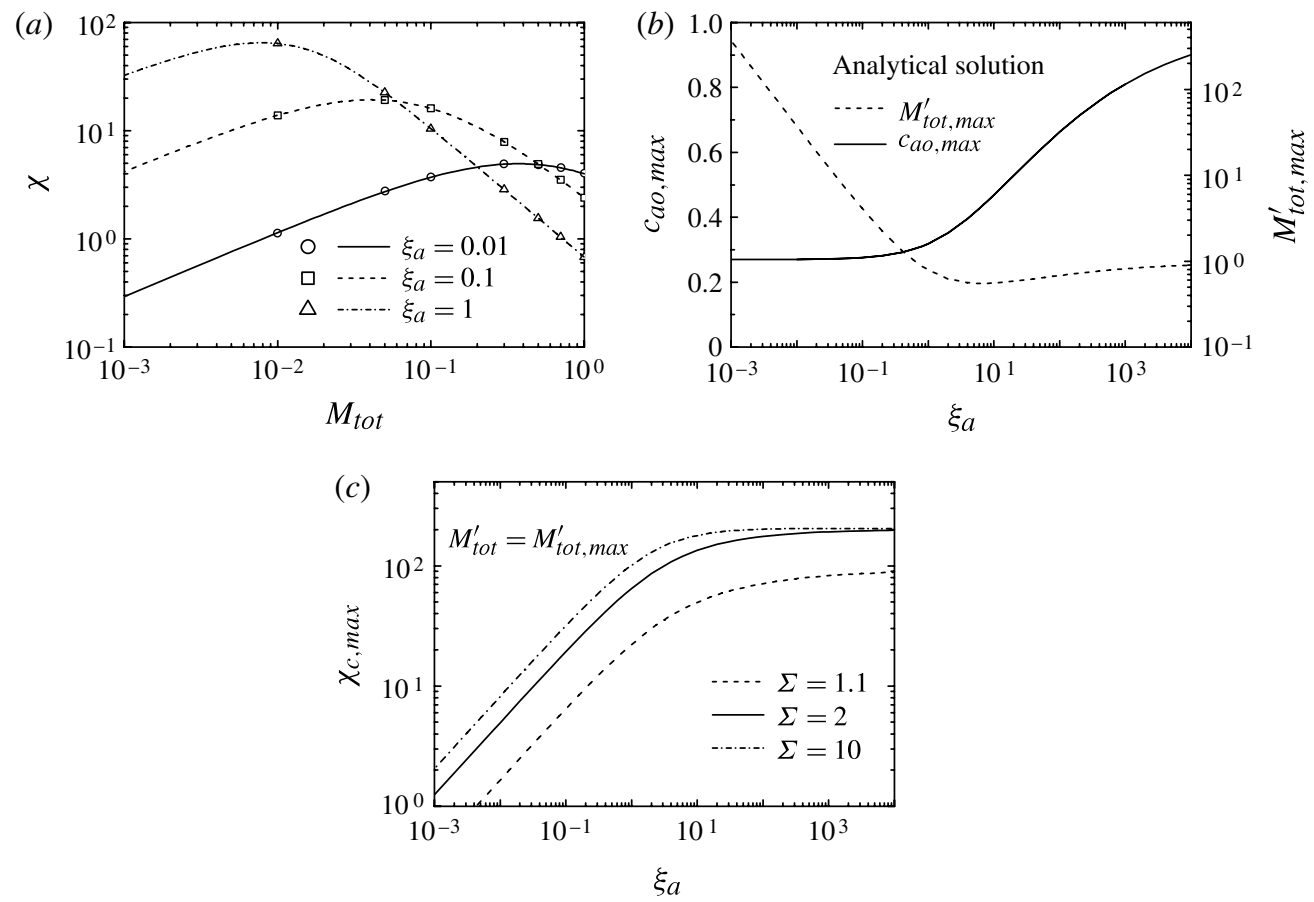

FIgURE 7. (a) Dependence of critical $\chi$ on $M_{\text {tot }}$; comparison of analytical solution (equation (4.42)) (lines) and numerical results (symbols) for $\alpha=90^{\circ}, \beta_{a}=0.01$ and $k=10^{-4}$. (b) Evaluation of $M_{t o t, \max }^{\prime}$ and $c_{a o, \max }$ for maximum stability of the flow as a function of $\xi_{a}$, using the analytical solution (equation (4.42)) for $\alpha=90^{\circ}$. (c) Evaluation of $\chi_{c, \max }$ for maximum stability of the flow as a function of $\xi_{a}$, using the analytical solution (equation (4.42)) for $\alpha=90^{\circ}$. The rest of the parameters remain the same as in figure 4 .

adsorbed surfactant at the liquid-air interface approaches saturation, surface tension gradients decrease and therefore the Marangoni effects become less significant. This is also reflected in (4.42) where the second term on the right-hand side is proportional to $c_{a o}-1$; for an interface saturated with a soluble surfactant ( $\xi_{a}$ not very large), the expression for the critical Reynolds reduces to the Newtonian limit.

The parametric variation of the curves in figure 7(a) with $\xi_{a}$ motivates investigation of two complementary issues: how does the location and how does the magnitude of the maximum in the critical conditions depend on surfactant solubility. Thus, in the following, we study $M_{t o t, \max }$ and $\chi_{c, \max }$ as a function of $\xi_{a}$. Starting with the former, we note that the value of $M_{t o t, \max }$ can be easily determined by solving the equation $\mathrm{d} \chi_{c} / \mathrm{d} M_{t o t}=0$, which for $\alpha=90^{\circ}$ and after some manipulation results in the following expression:

$$
8 E_{o}+\left(c_{a o}-1\right)\left[3 \xi_{a}\left(c_{a o}-1\right)^{2}+4\right] \frac{\mathrm{d} E_{o}}{\mathrm{~d} c_{a o}}=0 .
$$

The solution of (5.1) provides the surface concentration, $c_{a o, \max }$, that results in maximum stabilization, for a surfactant with solubility $\xi_{a}$. It is noteworthy that $c_{a o, \max }$ depends solely on $\xi_{a}$, and not independently on $\beta_{a}$ and $R_{a}$. According to the model adopted for surface elasticity, (4.26), $E_{o}$ is a function of $c_{a o}$ and $\Sigma$, so (5.1) needs to be solved numerically. 
The variation of the value of $c_{a o, \max }$ with surfactant solubility is shown in figure $7(b)$. For an insoluble surfactant $\left(\xi_{a} \rightarrow \infty\right)$, the flow is most stable with a saturated interface $\left(c_{a o} \rightarrow 1\right)$. This is readily explained by (4.44), in combination with the monotonic dependence of $E_{o}$ on $c_{a o}$. However, with increasing surfactant solubility, mass transfer between the bulk and the interface (leading to attenuation of surface tension gradients) grows in significance, and, as a result, maximum stabilization is achieved at lower values of $c_{a o, \max }$. For a highly soluble surfactant $\left(\xi_{a} \rightarrow 0\right)$, the most stable interfacial concentration reaches a plateau $c_{a o, \max } \approx 0.28$.

An alternative way of plotting the above result, also shown in figure $7(b)$, is in terms of the rescaled total amount of surfactant, $M_{t o t, \max }^{\prime}=M_{t o t, \max } / \beta_{a}$. This rescaling makes all data for different values of $\beta_{a}$ collapse onto the single curve shown. We note that $M_{t o t}$ and $M_{t o t}^{\prime}$ represent the total amount of surfactant, scaled respectively with the maximum capacity of the bulk and of the interface. An interesting observation from figure $7(b)$ is that the amount of surfactant, $M_{t o t, \max }^{\prime}$, corresponding to $\chi_{c, \max }$ is not a monotonic function of the solubility $\xi_{a}$; a minimum arises for moderate values of $\xi_{a}$, which indicates that optimum results may be achieved with smaller quantities of a slightly soluble surfactant than with an insoluble one.

The variation in the magnitude of the maximum, $\chi_{c, \max }$, with surfactant solubility is shown in figure $7(c)$ for three different values of parameter $\Sigma$, which is a measure of the strength of the surfactant and its ability to decrease the surface tension of the liquid-air interface. We note that $\chi_{c, \max }$ represents the optimum stabilization of the flow that can be achieved with surfactant of solubility $\xi_{a}$, and corresponds to the addition of amount $M_{\text {tot,max }}$. It is observed that, for soluble surfactants, $\chi_{c, \text { max }}$ increases with the decrease in solubility. However, below a moderate solubility, the curves reach an asymptotic limit, indicating that slightly soluble surfactants are equally efficient as insoluble ones.

An interesting observation from figure $7(c)$ is that there is a maximum stabilization that may be achieved, irrespective of the increase in $\Sigma$, which in the present case (and for $K a=3000$ ) is approximately $\chi_{c, \max } \leqslant 200$. This conclusion depends on the specific model used for surface tension variation. The stability problem for insoluble surfactant and a vertical film may be solved analytically with the present model, and gives the result

$$
\chi_{c, \text { insoluble }}=\left[\left(\frac{45}{2}\right) K a \frac{\Sigma^{1 / 3}-1}{\left[1+\left(\Sigma^{1 / 3}-1\right)\right]^{4}}\right]^{3 / 5} \quad \text { for } 1<\Sigma<64 / 27
$$

and

$$
\chi_{c, \text { insoluble }}=\left(\frac{1215}{512} K a\right)^{3 / 5} \text { for } \Sigma>64 / 27 .
$$

The above equations may be considered a special case of the general result for an insoluble surfactant (Whitaker 1964; Anshus \& Acrivos 1967),

$$
\chi_{c, \text { insoluble }}=\left(\frac{15}{2} K a E_{o}\right)^{3 / 5} \text {. }
$$

The aforementioned discussion, and the observation that the curves in figure $7(a)$ intersect, indicates that, for a specific total amount of surfactant, the dependence of critical conditions on surfactant solubility may be non-monotonic. Indeed, figure 8(a) shows the critical $\chi$ as a function of $\xi_{a}$ for different values of $R_{a}$ and for $M_{t o t}=0.1$. In all shown cases, $\chi_{c}$ exhibits a strong maximum at moderate values of $\xi_{a}$. Therefore, for a specific amount of surfactant, the moderately soluble one appears more efficient in stabilizing the flow than the insoluble one. Figure $8(b)$ shows again the dependence of 


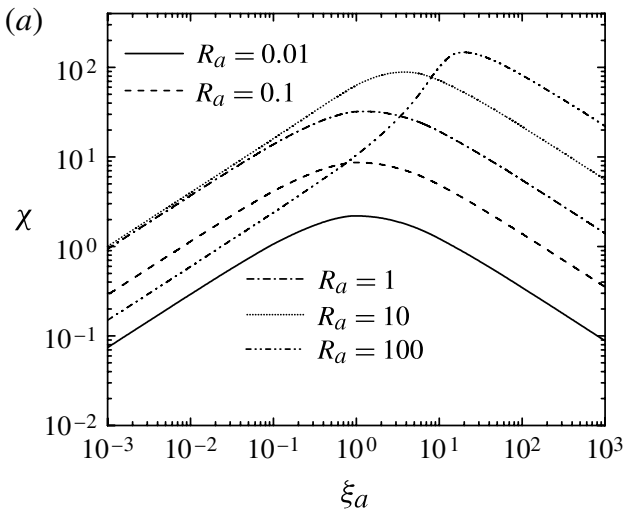

(b)

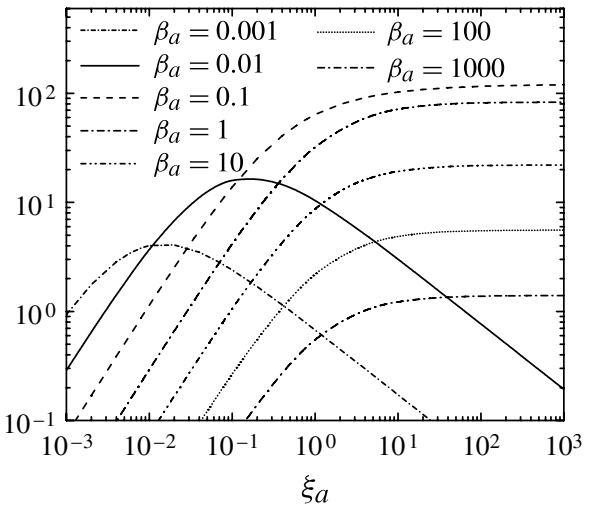

FIGURE 8 . Dependence of critical value of $\chi$ on the solubility parameter $\xi_{a}$ as predicted by the analytical solution (equation (4.42)) for different values of $R_{a}$ and for $\alpha=90^{\circ}, M_{t o t}=0.1$. The rest of the parameters remain the same as in figure 4, except for $\beta_{a}$ in figure $8(a)$ which varies as $\xi_{a} / R_{a}$, and for $R_{a}$ in figure $8(b)$ which varies as $\xi_{a} / \beta_{a}$.

critical $\chi$ on $\xi_{a}$, now varying the value of $\beta_{a}$. For small values of $\beta_{a}$, we observe again a non-monotonic dependence, whereas for larger values of $\beta_{a}$ the critical $\chi$ reaches a plateau in the limit of high $\xi_{a}$. This should come as no surprise because for large values of $\beta_{a}$ the capacity of the interface to adsorb monomers is very large and for the specific value of $M_{\text {tot }}$ the resulting interfacial concentration is very small (see also figure 2). The non-monotonic behaviour is recovered again when considering higher values of $M_{t o t}$.

\subsection{Parametric behaviour for disturbances of finite wavelength}

Having analysed the limit $k \rightarrow 0$, we now focus on the behaviour of disturbances of finite wavelength. The addition of one more length scale complicates significantly the dynamics. Among other effects, sorption kinetics - which is included in the modelling but did not appear in the limit of infinite wavelength - now becomes potentially important. Given the complexity of behaviour, we first perform an extensive parametric study and describe the results purely phenomenologically. Then, we sketch the key physical mechanisms that we believe determine the dynamics, and attempt some qualitative comparisons with available experimental observations.

\subsubsection{Effect of the total amount of surfactant}

Figure $9(a, b)$ shows the critical modified Reynolds number, $\chi$, as function of disturbance wavenumber for different amounts of surfactant, $M_{t o t}$. Figure $9(a)$ is a magnification of figure $9(b)$ at small values of $\chi$ and $k$. It is evident that the addition of a small amount of surfactant results in drastic stabilization of practically all disturbances of finite length.

In the small-wavenumber range, and with increasing amount of surfactant, the neutral curves in figure $9(a, b)$ tend to accumulate around a rough asymptote with slope $\approx 10^{4}$. The variation at higher wavenumbers is more complex, and eventually becomes non-monotonic. More specifically, an inflection point appears, which above $M_{\text {tot }}=0.3$ leads to a local maximum in $\chi$; a possible mechanism for this behaviour is described in $\S 5.4$, below. Further increase in the amount of surfactant renders higher wavenumbers progressively less stable. 

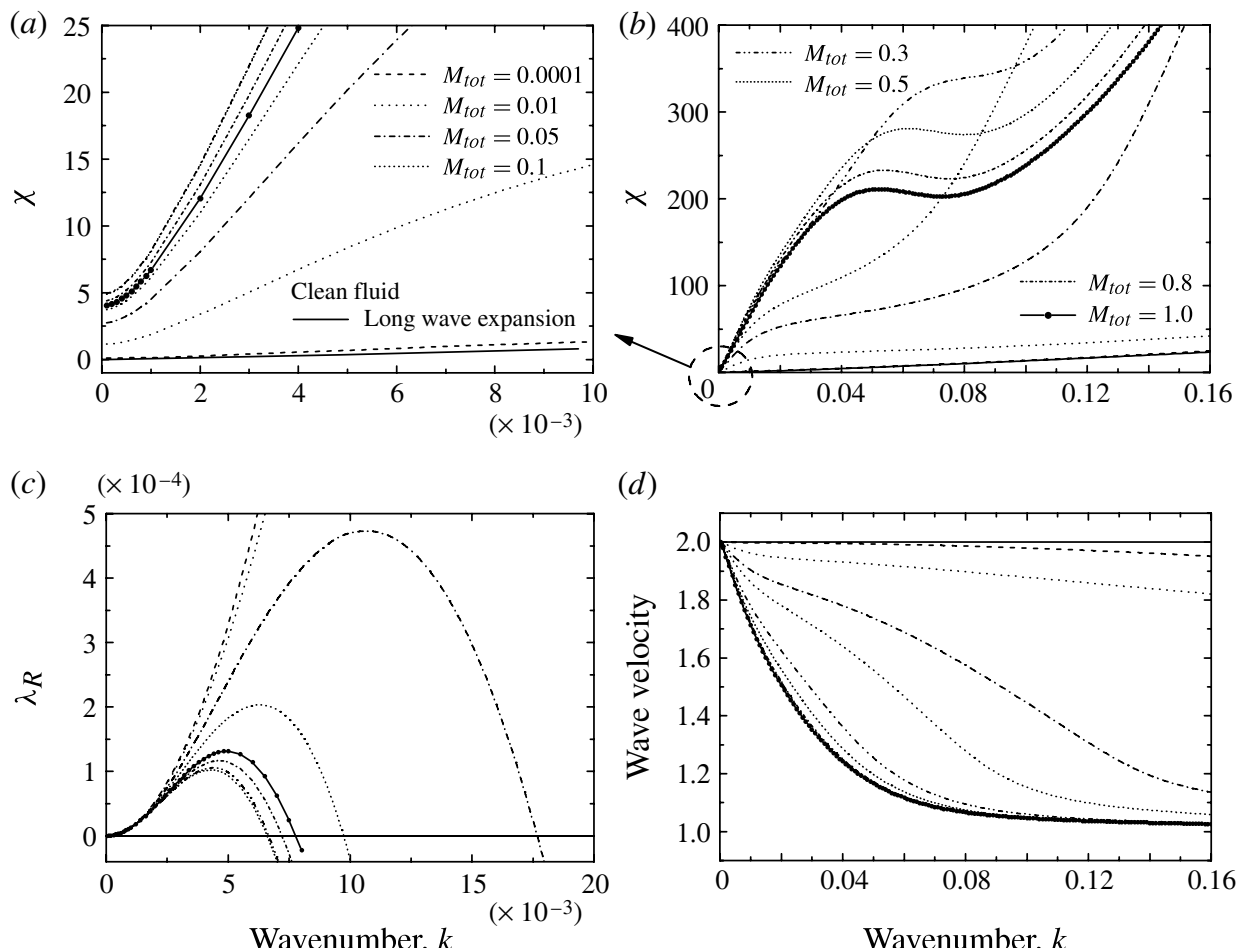

(d)

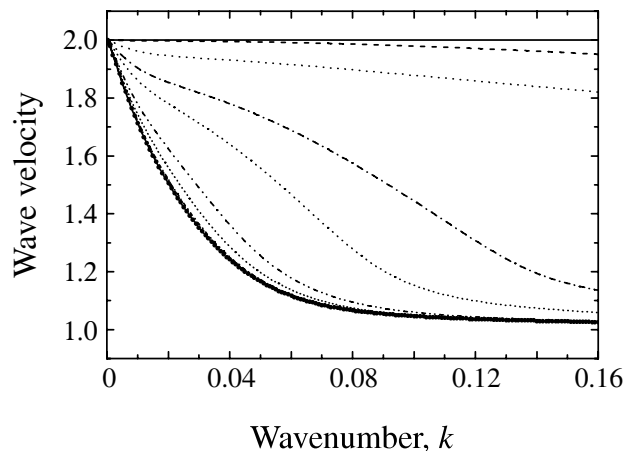

FIgURE 9. $(a, b)$ Neutral curves for different values of $M_{t o t}$; comparison with analytical solution for a clean fluid $\left(R e_{c}=5\left(\cot \alpha+W e k^{2}\right) / 4\right.$ or equivalently $\chi_{c}=5((\cot \alpha+$ $\left.\left.W e k^{2}\right) / \sin \alpha\right) / 2$ ); $(a)$ is a zoom of $(b)$ for $0<k<0.01$. (c) Dispersion curves for different values of $M_{t o t}$ and for $\chi=50$. $(d)$ Wave velocity of the marginally unstable mode at neutral stability as a function of the wavenumber, $k$, for different values of $M_{t o t}$. The rest of the parameters remain the same as in figure 4.

Figure $9(c)$ presents the dispersion curves for the same values of $M_{t o t}$ as in figure $9(a, b)$ and for a specific value of $\chi=50$. We observe that the addition of relatively small amounts of surfactant affects drastically the stability of the film, decreasing significantly the growth rate of the disturbances, as well as the range of the unstable modes. This result indicates that, in the presence of surfactants, instabilities will need considerably longer time to develop. The non-monotonic effect of $M_{t o t}$ that was discussed in figure 7 is also apparent here. For the given value of $\chi$, the growth rate of the disturbances (and in particular the most dangerous one) decreases for small values of $M_{t o t}$, reaches a minimum around $M_{t o t}=0.5$ and increases again for larger values of $M_{t o t}$. The behaviour of the wavenumbers of the most dangerous mode and of the cut-off is also similar.

Finally, figure $9(d)$ presents the wave velocity of the marginally unstable mode at neutral stability as a function of the wavenumber and for different amounts of surfactant. In agreement with previous experimental and theoretical studies (Strobel \& Whitaker 1969; Cerro \& Whitaker 1971), we find that the addition of surfactant decreases the wave velocity, and more so the higher the wavenumber. It is also notable that the variation with $M_{t o t}$ is strictly monotonic, reaching an asymptotic limit for high surfactant concentrations. Therefore, the wave velocity appears to depend directly on 

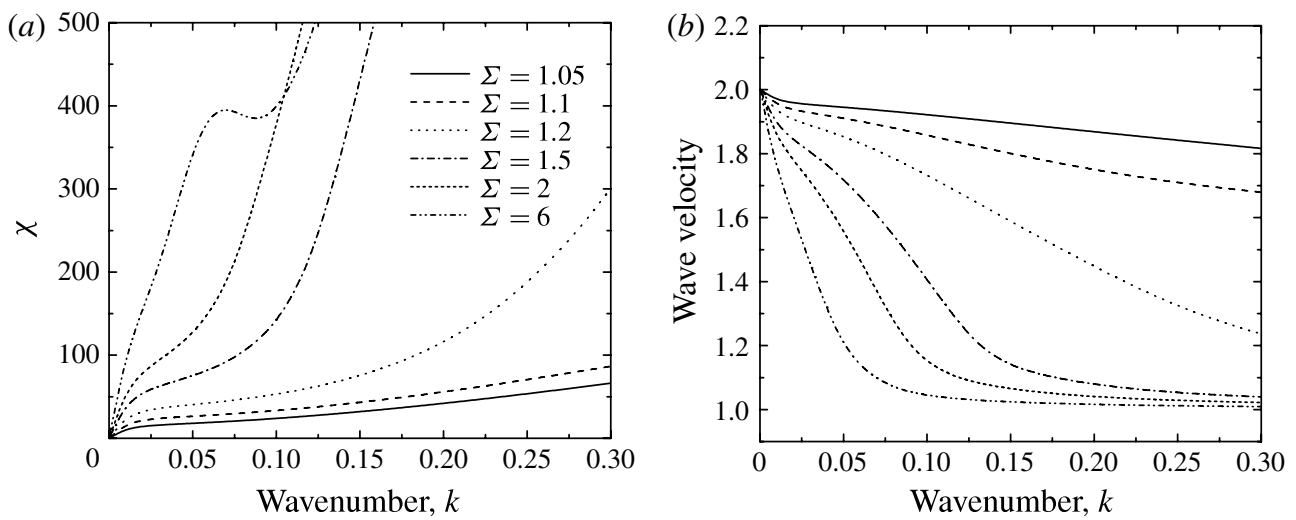

FIGURE 10. (a) Neutral curves and $(b)$ dependence of the wave velocity on the wavenumber for different values of $\Sigma$. The rest of the parameters remain the same as in figure 4 .

the surface concentration of the soluble surfactant, and not on the surface elasticity, as determined by the combination of stretching and diffusion/convection, which was discussed extensively in relation to figures 7 and 8 .

\subsubsection{Effect of surface tension and surfactant strength}

In figure 10(a) neutral curves are presented for various values of $\Sigma$ to examine the effect of Marangoni stresses. This parameter can be seen as a measure of the strength of the surfactant. Increasing $\Sigma$, while keeping $K a$ (i.e. $\sigma_{c}$ ) constant, means that the difference $\sigma_{c}-\sigma_{m}$ increases and therefore the surface tension gradients become stronger. The increase of the elasticity of the interface due to the induced Marangoni stresses stabilizes the flow and the critical $\chi_{c}$ increases. For low and moderate values of $\Sigma, \chi_{c}$ increases monotonically with the wavenumber of the disturbance. We find, though, that for large $\Sigma$ this is not always the case. Figure $10(b)$ shows the phase velocity of the marginally unstable mode, and indicates that it decreases drastically with the increase of $\Sigma$. In combination with figure $9(d)$, we conclude that the amount and the strength of the surfactant have a similar effect on the phase speed of the disturbances.

Figure 11 shows the effect of Kapitza number for a surfactant-laden liquid, along with the corresponding neutral curves of a clean liquid. Though the values of $K a$ plotted are high (and thus the effect of capillary forces on the stabilization of finitewavenumber disturbances in a clean liquid is significant), we note that, with the addition of surfactant, the critical $\chi$ rises by more than an order of magnitude. Another interesting observation is that the dependence of critical $\chi$ on $K a$ persists even for $k=0$ (see the insert of figure 11 for a zoom close to zero $k$ ), although it is well known that for a clean fluid and for $k=0$ the critical Reynolds number is independent of $K a\left(R e_{c}=5 \cot \alpha / 4\right)$. This observation is in agreement with the analytic result, see (4.42). One way to see why the value of $K a$ has such a strong impact on the stability of the surfactant-laden flow is by considering that increasing Kapitza number is like increasing the surface tension of the clean fluid, $\sigma_{c}$. For a given value of $\Sigma$ (in our case $\Sigma=2$ ) this would mean that the difference in surface tension between a clean interface and a fully contaminated one would increase proportionally and therefore the Marangoni effects would become more important. This trend is in agreement with theoretical studies in the literature for insoluble surfactants (Blyth \& Pozrikidis 2004; 


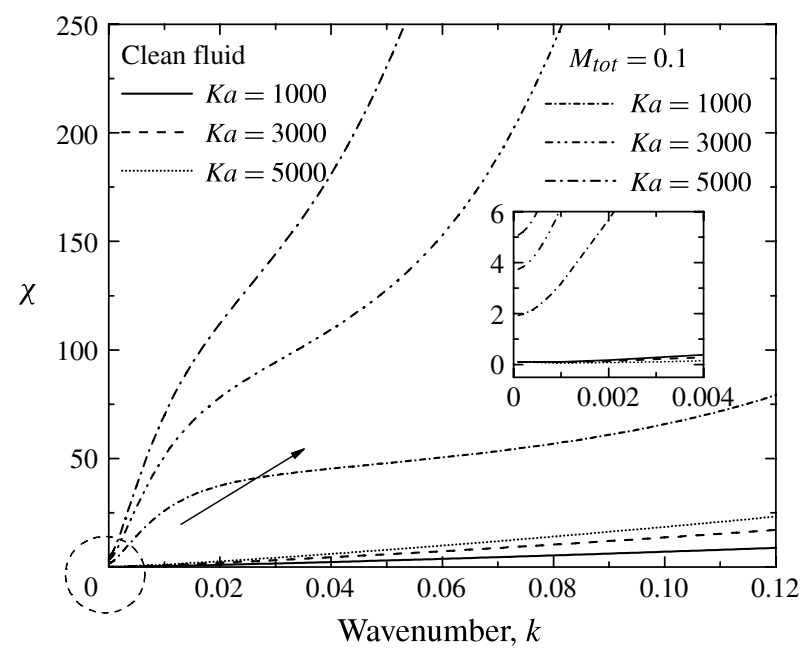

FIGURE 11. Neutral curves for different values of $K a$ for a surfactant-laden liquid, along with the corresponding neutral curves of a clean liquid. The rest of the parameters remain the same as in figure 4.

Pereira \& Kalliadasis 2008) where it was shown that the critical Reynolds number is proportional to the Marangoni number.

\subsubsection{Effect of surfactant solubility}

In figures 7 and 8 , we have examined the role of surfactant solubility, $\xi_{a}$, in the limit $k \rightarrow 0$. Now, we want to examine its effect for non-zero wavenumbers. To this end, we keep constant the total amount of surfactant $\left(M_{t o t}=0.1\right)$ and the value of $\beta_{a}$, which is a measure of the amount of surfactant that can be adsorbed at the interface, and vary the value of $\xi_{a}$ to examine the effect of surfactant solubility. For $\beta_{a}=0.01$ (shown in figure $12 a$ ), the effect of $\xi_{a}$ on critical $\chi$ appears to be rather complex for finite wavelengths. More specifically, we observe that for the lowest value of $\xi_{a}=0.001\left(R_{a}=0.1\right)$, the critical $\chi$ remains fairly constant for a wide range of wavenumbers, apart from a small region close to $k=0$. Increasing the value of $\xi_{a}$ to $0.01\left(R_{a}=1\right)$ the flow significantly stabilizes for short-wavelength disturbances. Further increase of $\xi_{a}$ may lead to situations where the system can be more stable to large-wavelength disturbances and at the same time less stable to short wavelengths (e.g. compare neutral curves for $\xi_{a}=0.01$ and $\xi_{a}=0.1$ ). For even higher values of the solubility parameter, $\xi_{a}$, the critical $\chi$ decreases significantly and for the highest value of $\xi_{a}$ the system behaves as a clean liquid; the neutral curves for $\xi_{a}=100$ and that of a clean fluid coincide, for the range of wavenumbers shown in the figure, but are not shown here for clarity. This happens because for $\xi_{a}=100$ (and for the specific amount of surfactant, $M_{t o t}=0.1$ ) the interface becomes saturated with surfactant (see also figure 2) and therefore the induced surface tension gradients are not important; this result is in agreement with the predictions of our analytical expression in the limit of $k \rightarrow 0$ (see (4.42) and relevant discussion above). For $\beta_{a}=1$ (shown in figure $12 b$ ) the picture is somewhat simpler, because the dependence of critical $\chi$ on $\xi_{a}$ is in this range monotonic. Nevertheless, we should note that the non-monotonic dependence is recovered again for higher values of $M_{t o t}$. 

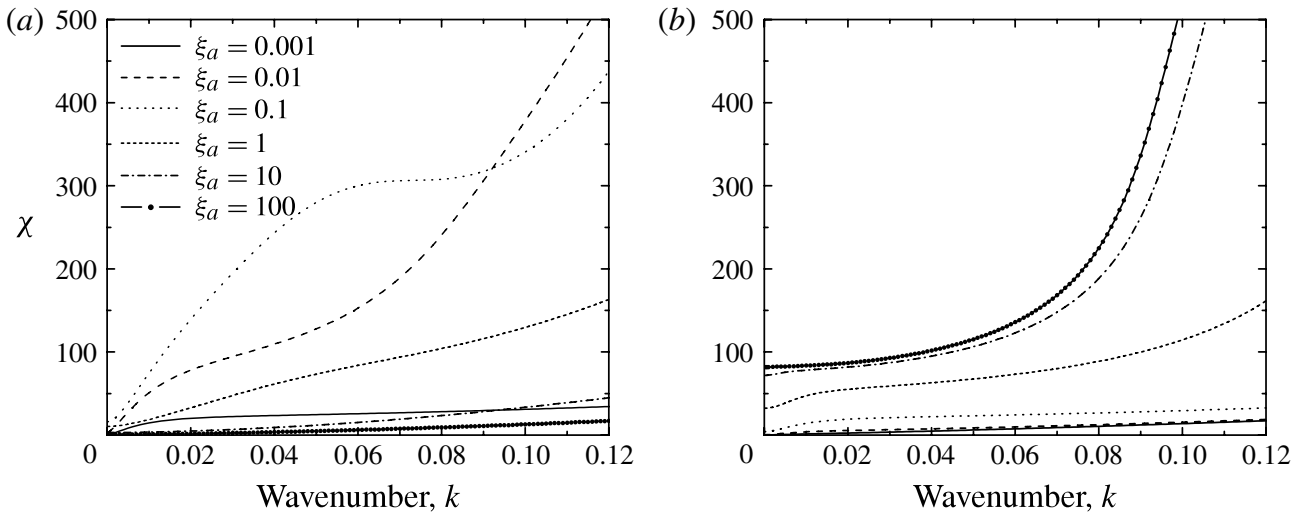

FIGURE 12. Neutral curves for different values of $\xi_{a}$ for $(a) \beta_{a}=0.01$ and $(b) \beta_{a}=1$. The rest of the parameters remain the same as in figure 4.

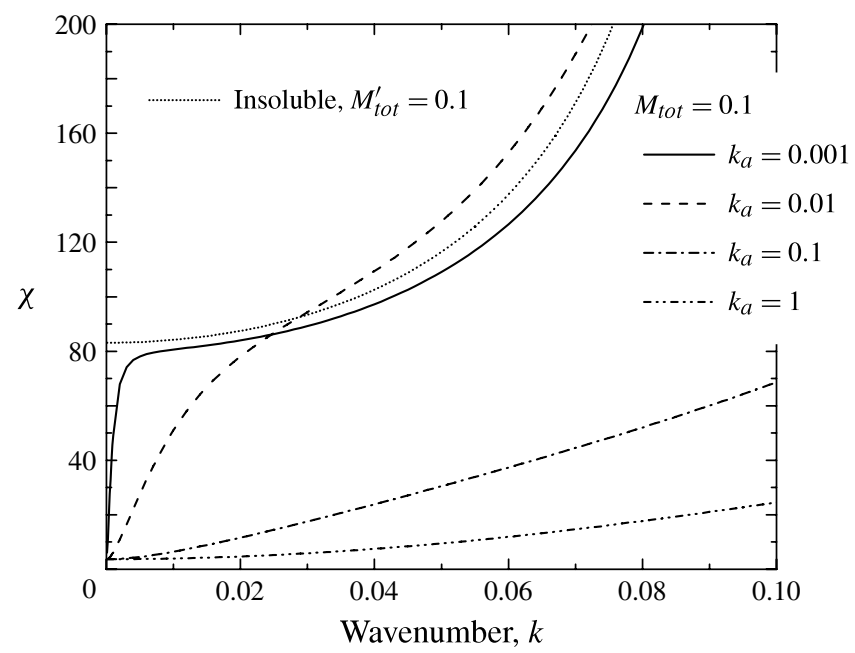

FIGURE 13. Neutral curves for different values of $k_{a}$. The rest of the parameters remain the same as in figure 4.

\subsubsection{Effect of sorption kinetics at the liquid-air interface}

Next, we consider the role of sorption kinetics at the liquid-air interface, which is potentially of central importance, and which has typically been neglected in simplified treatments of the problem. Thus, in figure 13 we examine the parametric effect of $k_{a}$ on the curves of neutral stability for the reference case of figure 4 . It is expected that for long-wave perturbations $(k \ll 1)$ the kinetics will not be very important because there will always be enough time for the different species to reach equilibrium around the interface. Indeed, as can be seen in figure 13, for $k=0$ the critical $\chi$ is the same for all values of $k_{a}$. This result is in direct agreement with the analytical expression that has been derived in the limit of $k \rightarrow 0$ (see (4.42)), where there is no dependence of the critical $R e$ on the value of $k_{a}$. 
However, we see that, for disturbances with larger wavenumbers, this is clearly not the case. In particular, we plot in figure 13 the result for an insoluble surfactant, and observe that, for the lowest value of $k_{a}$ shown, the neutral curve for the soluble surfactant follows it very closely, for all wavenumbers except for a very narrow range around zero. We should note at this point that to enable such a comparison between a soluble and an insoluble surfactant we have selected two cases with identical levels of interfacial concentration, $c_{a o}$. The similarity between the case of a soluble and an insoluble surfactant for large wavenumbers may be readily understood by considering that, when the kinetics are relatively slow and the disturbances have short wavelength, there will not be enough time for the species to approach equilibrium, and the system will behave as though the monomers that have been adsorbed at the liquid-air interface are isolated from the monomers in the bulk. The general trend with faster kinetics is for the soluble surfactant to become less stabilizing than the insoluble one for all wavenumbers. It is noteworthy, however, that there exists a range of moderate kinetics for which high-wavenumber disturbances are stabilized more efficiently with the soluble than with the insoluble surfactant (see $k_{a}=0.01$ and $k>0.03$ ).

\subsubsection{Effect of surfactant adsorption at the solid substrate}

Next we examine how the adsorption of surfactant at the solid substrate may affect the stability of the flow. To this end, we vary the relevant parameters that control the behaviour of the substrate. The effect of soprtion kinetics, $k_{s}$, is examined in figure $14(a)$ and shown to be negligible. On the contrary, the variation of $\beta_{s}$, which is shown in figure $14(b)$, appears to have a significant impact on the flow stability. These two figures indicate that the substrate acts as a surfactant reservoir, and its dynamics are relatively unimportant.

For the specific parameters that have been used in figure $14(b)$, increasing the value of $\beta_{s}$ (i.e. increasing the capacity of the substrate to adsorb surfactant) leads to stabilization of the flow. This behaviour may be understood by considering that the surfactant that adsorbs at the substrate is subtracted from the total amount residing in the bulk and at the liquid-gas interface. We already know, however, that in the absence of adsorption at the substrate, the stability of our system depends non-monotonically on the total amount of surfactant (see figure 7 and the relevant discussion for the effect of $M_{t o t}$ ). Since this total amount of surfactant varies with the adsorbability of the substrate, we deduce that different substrates may also affect the flow stability in a non-monotonic way.

This is confirmed in figure $14(c)$, where we have plotted the critical $\chi$ as a function of the parameter $\beta_{s}$. For all values of $\xi_{s}$ (which expresses the relative solubility in the bulk with respect to adsorption on the substrate), the flow acquires maximum stability for intermediate values of $\beta_{s}$. For low values of $\beta_{s}$, critical $\chi$ tends to the limit of no adsorption at the substrate, whereas for high $\beta_{s}$ the capacity of the substrate to adsorb surfactant increases drastically and this leads to a decrease of $\chi_{c}$ as a large amount of surfactant is essentially removed from the system. It is noted in particular, that the decline of $\chi_{c}$ occurs at roughly constant $\beta_{s}\left(\beta_{s} \approx 10^{-1}\right)$, and is steeper the higher the value of $\xi_{s}$. These observations indicate that, when surfactants are to be used in order to stabilize the flow in various applications, it is also very important to pay special attention to the affinity of the selected surfactant with the substrate in hand.

\subsubsection{Effect of surfactant diffusivity}

Finally, figure 15 presents neutral curves for various values of the Schmidt number $S c_{a}$ and $S c_{b}$. It is evident that the effect of diffusion does not play any important role in the stability of the flow. 

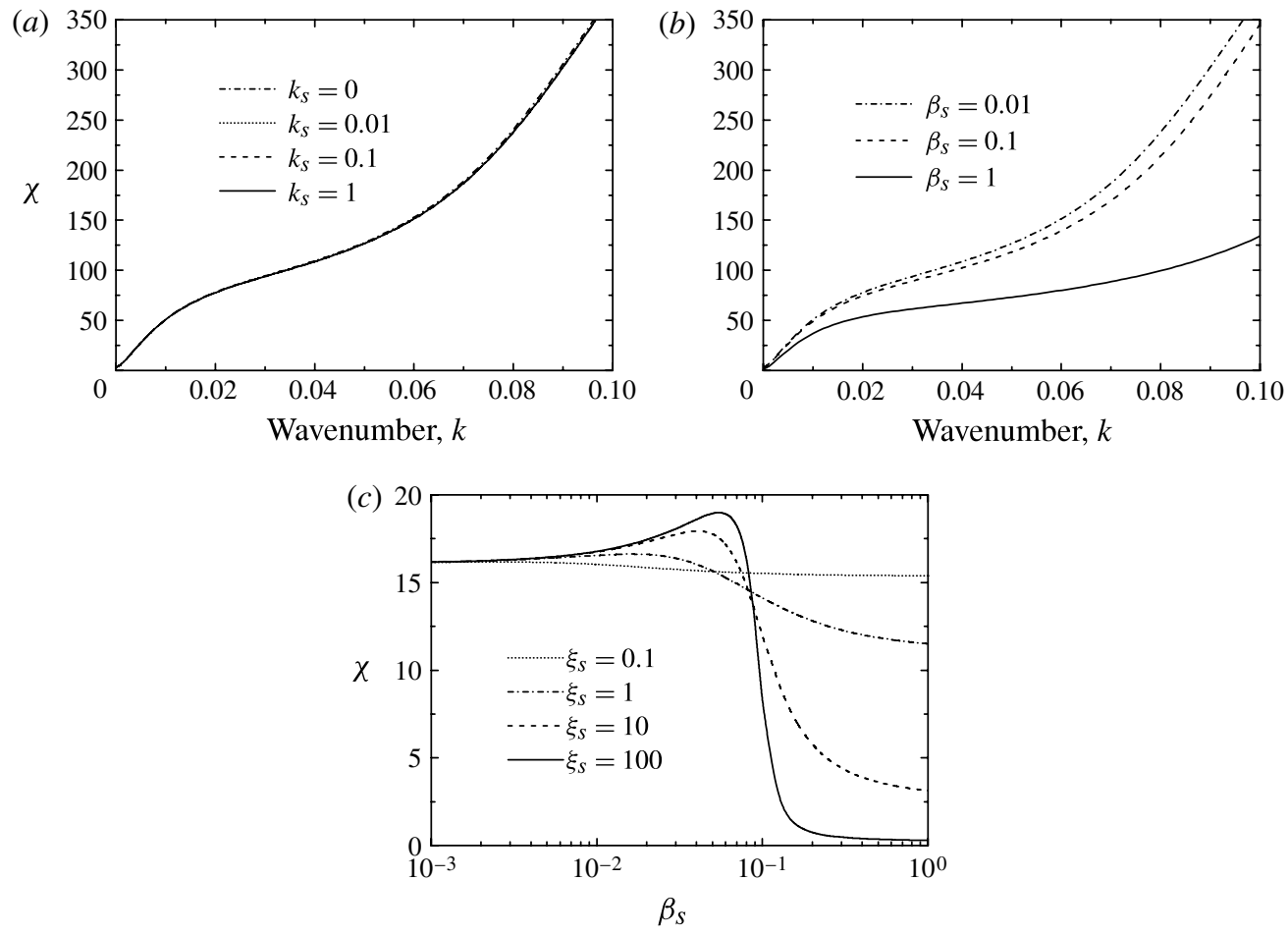

FIGURE 14. Effect of the adsorption of surfactant at the substrate. Neutral curves for $R_{s}=1$ and various values of $(a) k_{s}$ for $\beta_{s}=0.01, R_{s}=1$ and $(b) \beta_{s}$ for $k_{s}=0.1, R_{s}=1$. (c) Critical $\chi$ as a function of $\beta_{s}$ for various $\xi_{s}$ for $k_{s}=0.1$. The rest of the parameters remain the same as in figure 4 .
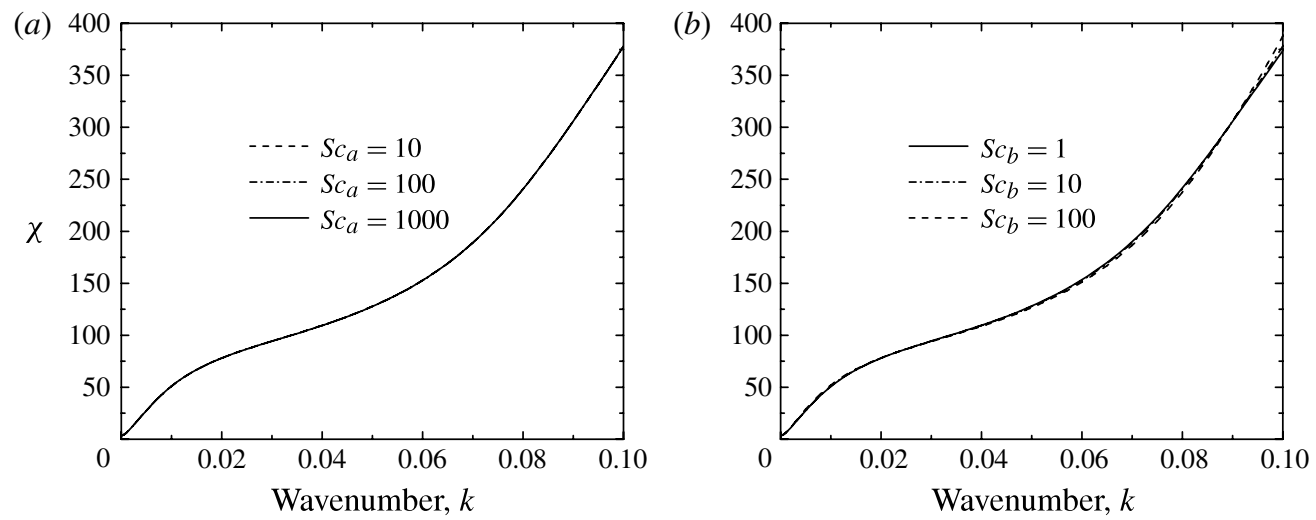

FIgURE 15. Neutral curves for different values of (a) $S c_{a}$ and (b) $S c_{b}$. The rest of the parameters remain the same as in figure 4.

\subsection{Postulation of mechanisms and interpretation of experiments}

We conclude the above presentation of the effect of various system parameters with a discussion of possible mechanisms that operate in the case of soluble surfactants. Then, we attempt to interpret recent experimental observations that motivated this study, in 
the light of the present findings. With respect to potential mechanisms, we identify four key components: (i) The effect of solubility in enhancing mass exchange between the interface and the bulk. It appears that this mechanism determines the critical conditions that correspond to very long waves, and may lead to optimal behaviour of moderately soluble surfactants. (ii) The direct effect of disturbance wavenumber. With increasing wavenumber, gradients in surface concentration of surfactant are expected to intensify, because variations take place on a shorter length scale. In addition to this, mass exchange - which mitigates gradients according to (i) - is weakened, because there is less time for the approach to equilibrium. (iii) The effect of disturbance phase velocity. We have observed that surfactant decreases drastically the phase velocity at high wavenumbers. Though we do not have a full understanding of the mechanism by which the flow stabilizes, we may plausibly assume that, as the wave moves downstream, it continuously sweeps surfactant by convection, thus inducing stabilizing surface tension gradients. This effect is expected to weaken with the decrease in phase velocity. The above components (ii) and (iii), that operate at high wavenumbers, have a competing effect, and their interplay could be responsible for the non-monotonic dependence of the critical conditions on wavenumber, which was observed at high amount/strength of surfactant (see figures $9 b$ and 10a). (iv) The effect of phase shift between disturbances at the interface. An understanding of the interaction between disturbances in height, velocity, surface concentration and shear stress would probably help unify the above components and delineate the complete stabilization mechanism. Work will be undertaken in this direction, following the approach of Smith (1990).

Returning to the experiments by Georgantaki et al. $(2011,2012)$, and in particular to the effect of soluble surfactant SDS, we note the following: strong stabilization of liquid film flow was observed with the addition of small amounts of SDS, and the effect maximized at concentrations of the order of $10 \%$ of the critical micelle concentration, and then gradually reduced. This behaviour agrees qualitatively with the prediction for the critical conditions of a moderately soluble surfactant, as exhibited for example by figure $7(a)$.

The dominant structures observed, even deep in the unstable regime, were sinusoidal travelling waves of small amplitude. Only at very high $R e$ in combination with very low-frequency inlet forcing, were non-sinusoidal structures observed, and these differed in shape from the solitary waves encountered in clean liquids. These observations are in accord with the predictions of the present study. In particular, it was shown that small amounts of soluble surfactant decrease drastically both the range and the growth rate of unstable wavenumbers (figure 9a,c). Smaller growth rate means that energy input from the mean flow to the unstable wavenumbers will be slower. This effect will further intensify, as the experimental inlet frequency remains constant while the cut-off frequency decreases. In addition, higher harmonics (which are sustained by energy input from the dominant mode through nonlinear coupling, and which contribute to the shape of the final free-surface structures) have typical amplitudes of order inversely proportional to their damping rates. Thus, with the displacement of the entire dispersion curve to lower values, the magnitude of higher harmonics declines as well. The combination of all these effects explains the experimentally observed persistence of nearly sinusoidal waves.

\section{Conclusions}

We have investigated the linear stability of a film flowing down a solid substrate in the presence of a soluble surfactant. We used a detailed surfactant model, which 
considers monomers dissolved in the bulk and adsorbed with Langmuir kinetics at the gas/liquid and the solid/liquid interfaces. The Navier-Stokes equations for the liquid motion and the advection-diffusion equations for surfactant concentrations were linearized around the base flow, resulting in an Orr-Sommerfeld eigenvalue problem that was solved analytically in the limit of long-wave disturbances, and numerically for arbitrary wavelength using a finite element method.

The instability was shown to be a long-wave one, and the derived analytic solution for $k \rightarrow 0$ indicated that, among all the model parameters, critical conditions depend only on the solubility of the surfactant and on its interfacial concentration. An interesting finding was that, for a given total amount of surfactant, a moderately soluble one may produce stronger stabilization than the insoluble one. Optimum conditions were also found to vary with solubility, the insoluble surfactant maximizing its performance at the tightest interfacial packing, whereas the soluble one did so at a fraction of it. These observations were explained by the competing effect of mass exchange between the interface and the bulk, which short-circuits surface tension gradients, and thus attenuates Marangoni stresses.

Disturbances of finite wavelength were considered next, and the additional length scale was observed to cause an escalation in complexity. Small amounts of soluble surfactant stabilize drastically the entire spectrum by shrinking the unstable range of wavenumbers, and by decreasing their growth rate and phase velocity. However, the effect maximizes at intermediate amounts and then drops again. For a fixed amount of surfactant, the role of solubility was shown to be highly non-trivial, and to depend strongly on the relative capacity of the interface and the bulk, $\beta_{a}$. Sorption kinetics at the gas-liquid interface was found to play a key role at finite wavelengths, with very slow kinetics leading to a virtually frozen interface and an insoluble-like behaviour. Finally, it was shown that the adsorption capacity of the solid substrate may also have a significant effect.

In an attempt towards mechanistic understanding of the stabilization offered by a soluble surfactant, some factors contributing to the magnitude of interfacial gradients of surfactant concentration were proposed. These include mass exchange with the bulk, and wavelength and phase velocity of travelling disturbances. Finally, predictions by the present study appear to interpret convincingly some recent experimental observations on the effect of the soluble surfactant SDS.

\section{Acknowledgements}

The authors would like to acknowledge the financial support by the General Secretariat of Research and Technology of Greece under the Action 'Supporting Postdoctoral Researchers' (grant number: PE8/906), co-funded by the European Social Fund and National Resources and to thank the referees for many insightful comments that improved significantly the quality of the manuscript.

\section{REFERENCES}

Anshus, B. E. \& ACRIVOS, A. 1967 The effect of surface active agents on the stability characteristics of falling liquid films. Chem. Engng Sci. 22 (3), 389-393.

Benjamin, T. B. 1957 Wave formation in laminar flow down an inclined plane. J. Fluid Mech. 2, 554-573.

Benjamin, T. B. 1964 Effects of surface contamination on wave formation in falling liquid films. Arch. Mech. Stos. 16, 615.

Blyth, M. G. \& PozRikidis, C. 2004 Effect of surfactant on the stability of film flow down an inclined plane. J. Fluid Mech. 521, 241-250. 
CErro, R. L. \& Whitaker, S. 1971 Entrance region flows with a free surface: the falling liquid film. Chem. Engng Sci. 26 (6), 785-798.

Chang, H. 1994 Wave evolution on a falling film. Annu. Rev. Fluid Mech. 26, 103-136.

Craster, R. V. \& Matar, O. K. 2009 Dynamics and stability of thin liquid films. Rev. Mod. Phys. 81 (3), 1131.

Edmonstone, B. D., Craster, R. V. \& Matar, O. K. 2006 Surfactant-induced fingering phenomena beyond the critical micelle concentration. J. Fluid Mech. 564, 105-138.

EDWARDs, D. A., BREnNER, H. \& WASAN, D. T. 1991 Interfacial Transport Processes and Rheology. Butterworth-Heinemann.

Emmert, R. E. \& Pigford, R. L. 1954 A study of gas absorption in falling liquid films. Chem. Engng Prog. 87-93.

Fruhner, H., WAntKe, K.-D. \& Lunkenheimer, K. 1999 Relationship between surface dilational properties and foam stability. Colloids Surf. A: 162, 193-202.

Gaver, D. P. \& Grotberg, J. B. 1990 The dynamics of a localized surfactant on a thin film. J. Fluid Mech. 213, 127-148.

Georgantaki, A., Vatteville, J., Vlachogiannis, M. \& Bontozoglou, V. 2011 Measurements of liquid film flow as a function of fluid properties and channel width: evidence for surface-tension-induced long-range transverse coherence. Phys. Rev. E 84, 026325.

Georgantaki, A., Vlachogiannis, M. \& Bontozoglou, V. 2012 The effect of soluble surfactants on liquid film flow. J. Phys. Conf. Ser. 395 (1), 012165.

Jensen, O. E. \& Grotberg, J. B. 1993 The spreading of heat or soluble surfactant along a thin liquid film. Phys. Fluids A 5 (1), 58.

Ji, W. \& Setterwall, F. 1994 On the instabilities of vertical falling liquid films in the presence of surface-active solute. J. Fluid Mech. 278, 297-323.

Karapetsas, G., Craster, R. V. \& Matar, O. K. $2011 a$ On surfactant-enhanced spreading and superspreading of liquid drops on solid surfaces. J. Fluid Mech. 670, 5-37.

Karapetsas, G., Craster, R. V. \& Matar, O. K. $2011 b$ Surfactant-driven dynamics of liquid lenses. Phys. Fluids 23 (12), 122106.

LIN, S. P. 1970 Stabilizing effects of surface-active agents on a film flow. AIChE J. 16 (3), 375-379.

Oron, A., Davis, S. H \& Bankoff, S. G 1997 Long-scale evolution of thin liquid films. Rev. Mod. Phys. 69 (3), 931-980.

Pereira, A. \& Kalliadasis, S. 2008 Dynamics of a falling film with solutal Marangoni effect. Phys. Rev. E 78 (3), 036312.

POZRIKIDIS, C. 2003 Effect of surfactants on film flow down a periodic wall. J. Fluid Mech. 496, 105-127.

Sheludko, A. 1967 Thin liquid films. Adv. Colloid Interface Sci. 1, 391-464.

Shkadov, V. Y., Velarde, M. G. \& Shkadova, V. P. 2004 Falling films and the Marangoni effect. Phys. Rev. E 69 (5), 056310.

Sмiтh, M. K. 1990 The mechanism for the long-wave instability in thin liquid films. J. Fluid Mech. 217, 469-485.

Stirba, C. \& Hurt, D. M. 1955 Turbulence in falling liquid films. AIChE J. 1 (2), 178-184.

Strobel, W. J. \& WhitAKer, S. 1969 The effect of surfactants on the flow characteristics of falling liquid films. AIChE J. 15 (4), 527-532.

TAilby, S. R. \& Portalski, S. 1961 The optimum concentration of surface active agents for the suppression of ripples. Trans. Inst. Chem. 39, 328-336.

WhitAker, S. 1964 Effect of surface active agents on the stability of falling liquid films. Ind. Engng Chem. Fundam. 3 (2), 132-142.

Whitaker, S. \& Jones, L. O. 1966 Stability of falling liquid films. Effect of interface and interfacial mass transport. AIChE J. 12 (3), 421-431.

Yiantsios, S. G. \& Higgins, B. G. 2010 A mechanism of Marangoni instability in evaporating thin liquid films due to soluble surfactant. Phys. Fluids 22, 022102.

YIH, C.-S. 1963 Stability of liquid flow down an inclined plane. Phys. Fluids 6, 321-334. 